\title{
Internet e Historia contemporánea de España
}

\author{
Ángel Martínez de Velasco Farinós
}

Internet es un término de origen informático del que poco se sabe aunque mucho se habla; tanto que ha llegado a ser un auténtico mito sociocultural. En este artículo se intenta explicar la joven historia de Internet como medio para llegar a la descripción de los servicios que pueden ser útiles para los historiadores. Posteriormente se muestra la aplicación de Internet hecha para el Departamento de Historia Contemporánea de la UNED y finaliza con un breve comentario bibliográfico *.

\section{HISTORIA DE INTERNET}

Hace más de treinta años la posibilidad de un ataque nuclear era un supuesto manejado por las autoridades norteamericanas inmersas en plena psicosis de la guerra fría '. El gabinete de estrategia de la empresa RAND se planteó el siguiente problema: ¿cómo se podrían comunicar las autoridades norteamericanas después de una guerra nuclear? La Norteamérica postnuclear necesitaría una red robusta de mando y control que uniera entre sí las bases militares esparcidas por todo el país. Sin embargo, por mucho que se protegieran o blindaran los nodos ${ }^{2}$ y las líneas de comunicaciones, siempre sería vulnerable al impacto de las bombas atómicas que reducirian a jirones cualquier red de las entonces existentes.

* Este articulo se finalizó en agosto de 1995.

- Para la elaboración de este apartado se ha seguido el articulo de BruCE Sterling, titulado "internet" y publicado en The Magazine Of Fantasy And Science Fiction, Febrero 1993, Cornwall, CT 06753. José BARBERA, Director del Departamento de Redes de FUNDESCO, en su artículo "Veinticinco años de Internet: una retrospectiva autobiográfica", publicado en el número 32 del Boletin de la red nacional de 1+D, Red IRIS, 23-34, utiliza fundamentaimente el de STERLING.

2 Un nodo es un ordenador principal dentro de una red. 
Cualquier autoridad y cualquier ciudad donde se encontrase la central de la red serian los objetivos inmediatos y obvios para los misiles enemigos.

Tras largas elucubraciones el gabinete de estrategia de la RAND, dirigido por Paul Baran, hizo pública en 1964 una propuesta encaminada a combatir esa eventualidad. En primer lugar, tal red no tendría una autoridad central; además habría que diseñarla desde el principio para que pudiera operar siempre, aunque sólo quedasen jirones de ella, de modo que la destrucción de una parte no afectase al resto de los nodos. Así pues, se partía de que la red no sería nunca jerárquica y de que todos los nodos debían ser iguales, con la misma autoridad para enviar, pasar y recibir los mensajes.

Estos mensajes se dividirían en "paquetes", cada uno de ellos con su propia identidad, que se enviarían separadamente. Cada paquete comenzaría en algún nodo de origen y serpentearía por la red hasta que alcanzase su nodo de destino. El camino particular que llevase cada paquete no importaba, sólo contaba el resultado final, es decir, que existieran los mecanismos adecuados para recomponer el mensaje original en el lugar de destino. En esencia, cada paquete iría saltando de nodo en nodo como una patata caliente, más o menos en dirección a su destino. Si en el camino algún nodo fallaba, los paquetes quedaban como flotando en el aire, dirigiéndose a otros nodos vecinos que les permitirían sobrevivir. Nacía de este modo el concepto de red de conmutación de paquetes, un sistema de comunicación que podría ser dudosamente eficaz desde el punto de vista convencional (sobre todo si se compara con la red telefónica ordinaria), pero que sería enormemente robusto frente a adversidades y fallos.

La idea de crear una red a prueba de bombas, descentralizada y basada en la conmutación de paquetes, fue tomando cuerpo y encontró eco entre los investigadores de la RAND, del Instituto Tecnológico de Massachusetts (MIT) y de la Universidad de California de Los Ángeles (UCLA). El Laboratorio Nacional de Física del Reino Unido construyó un prototipo de red con esas características en 1968 y poco después la Agencia de Proyectos de Investigación Avanzada (ARPA) del Ministerio de Defensa norteamericano decidió subvencionar un experimento similar, aunque más ambicioso, en el que los nodos de la red serían las mejores supercomputadoras que entonces existían y que debían de servir para la investigación nacional y el desarrollo de proyectos militares.

A finales de 1969 se instaló y puso en funcionamiento el primer nodo en la UCLA. En diciembre ya había otros tres más: en el Instituto de Investigación de Stanford (SRI), en la Universidad de California en Santa Bárbara (UCSB) y en la Universidad de Utah. Estos cuatro nodos, conocidos 
por las siglas IMP (Inteface Message Processor), formaron una red llamada ARPANET al estar financiada por el Pentágono. Las cuatro computadoras transferían sus datos a través de líneas exclusivas de alta velocidad $(56.000$ bites por segundo $056 \mathrm{Kbps}$ ) y los científicos e investigadores de estas instituciones pudieron compartir información e incluso programar remotamente los ordenadores. Todo esto resultaba particularmente interesante en aquellos años en los que el tiempo de utilización de las máquinas era un recurso especialmente valioso.

En 1971 habia quince nodos en ARPANET y en 1972, treinta y siete. Se constituía de ese modo el embrión de lo que hoy es Internet. El tiempo demostró que la realización de la idea de Paul Baran era factible y utilísima. Sin embargo, durante el segundo año de funcionamiento, apareció un hecho no previsto inicialmente al diseñar el proyecto. La utilización principal de ARPANET por los científicos no era la de programar remotamente los superordenadores, sino la de intercambiar mensajes sobre los trabajos en curso, notas comerciales e incluso chismes y cotilleos. Los usuarios habian convertido así la red de recursos informáticos compartidos en un servicio de comunicación personal, el correo "electrónico", que era más económico que el correo normal, -estaba subvencionado con fondos públicos-, y más interesante que el frío diálogo remoto con las máquinas.

El paso siguiente fue el descubrimiento de las listas de distribución electrónicas, que permitían difundir un mismo mensaje a un gran número de personas interesadas sobre un mismo tema. Uno de los más populares fue el foro de discusión dedicado a la ciencia ficción; tema que no tenía que ver demasiado con el objetivo del proyecto y que en cierta medida contrarió a los responsables de ARPA. Lo que inicialmente se concibió como una red de ordenadores para comunicarlos entre sí, se había transformado en una red para la comunicación de personas y grupos, basada en ordenadores y en la conmutación de paquetes.

Durante los años 70 la red ARPA fue creciendo gracias a su estructura descentralizada. A diferencia de las redes de ordenadores corporativas que eran homogéneas, ARPANET admitía sistemas diferentes con tal de que cumplieran una condición imprescindible: todos debian hablar un mismo lenguaje discontinuo NCP (Network Control Protocol). Mientras tanto iban desarrollándose proyectos de redes de conmutación de paquetes basadas en otras tecnologías y medios de transmisión como la radio y el satélite ${ }^{3}$. A

3 Posteriormente esta tecnologia dio origen a la red Ethernet desarrollada en el centro de investigación de Xerox en Palo Alto. 
la vista de esa situación, en 1973 los responsables de ARPANET lanzaron una nueva iniciativa con el objetivo de investigar técnicas y tecnologías para unir redes de paquetes de varios tipos. La idea, bautizada como Proyecto Interneting, del que ha derivado el nombre actual de Internet, era desarrollar unas normas, llamadas protocolos de comunicación que permitieran a los ordenadores comunicarse de modo transparente a través de distintas redes de paquetes interconectadas ${ }^{4}$.

El sistema de protocolos desarrollado en el curso de ese proyecto es lo que se conoce como la serie de protocolos TCP/IP, por los acrónimos de los dos primeros: Transmission Control Protocol (TCP) e Internet Protocol (IP). El primero de ellos trocea en paquetes los mensajes generados en el origen, y luego los recompone en el nodo de destino. El Protocolo IP se ocupa de dirigir los paquetes, de modo que éstos puedan viajar por rutas diversas, atravesando múltiples nodos e incluso por diferentes redes con distintos estándares de comunicación.

A comienzos de la década de los 80 surgen en el mundo científico de EE.UU. otras redes afines, tales como la CSNET y BITNET 5 . Mientras tanto el uso de los protocolos TCP/IP se fue generalizando en otras redes para su conexión a ARPANET, que seguia creciendo de forma sostenida, aunque en 1983 se desgajó de la parte relacionada con la defensa, que recibió el nombre de MILNET. A pesar de su crecimiento, ARPANET fue quedando como una comunidad más reducida frente a otras que iban surgiendo, impulsadas por la necesidad de conectar las nuevas y potentes máquinas que comenzaban a proliferar. Estas máquinas se interconectaban entre sí mediante los protocolos TCP/IP, que actuaban como un pegamento transparente que unía múltiples redes sin costuras aparentes. Puesto que el software TCPIIP era de dominio público y la tecnología básica era descentralizada -y más bien anárquica-, era imposible frenar el

4 Un protocolo es una definición de cómo se comportan dos ordenadores cuando se comunican entre si. Las definiciones de protocolo van desde la colocación de los bits en el medio de trasmisión hasta el formato de un mensaje de correo electrónico. Los protocolos estándares permiten que ordenadores de diferentes fabricantes puedan comunicarse; los ordenadores pueden usar software de distintos fabricantes y distintas presentaciones siempre y cuando ambas estén de acuerdo con el significado de la información.

5 La CSNET (Computer Science Net) fue en un principio patrocinada por la National Science Foundation (NSF) para unir grupos de investigación en ciencia informática en universidades, centros públicos e industria. Inicialmente usaba el protocolo MMDF Phonenet como base para el correo electrónico sobre líneas telefónicas; es de destacar que fue la primera red que posteriormente experimentó ef uso del TCP/IP sobre X.25 en redes públicas de datos. La red BITNET, de naturaleza interdisciplinar, conectaba los ordenadores IBM de los centros de cálculo de diversas universidades con líneas de baja velocidad mediante la seríe de los primitivos protocolos RSCS de IBM. 
impulso de interconexión de los usuarios. De hecho nadie quería impedir la interconexión de todas esas redes. De este modo, nació en $1983 \mathrm{IN}$ TERNET como red de interconexión entre ARPANET, MILNET y CSNET, unidas todas ellas por los protocolos TCP/IP, y a las que se irian añadiendo posteriormente otras redes de EE.UU. y de otros países.

En 1986 se puso en marcha una nueva iniciativa para unir los nodos principales mediante líneas de alta velocidad, creando de este modo una red troncal (backbone) con enlaces cuya capacidad originaria era de un millón y medio de bites por segundo (1,5 Mbps) y que actualmente han alcanzado los 100 Mbps. Paralelamente, algunas agencias del gobjerno norteamericano, (la NASA, el Departamento de Energía, el Instituto Nacional de la Salud, etc.), fueron poniendo en marcha sus propias redes, creando de este modo una confederación Internet, con una estructura organizativa descentralizada propia de los reinos de taifas.

A principios de los 90 se produce un giro estratégico en la concepción de la red, pues empezaron a introducirse empresas que empleaban Internet para sus propios fines comerciales, de forma que los beneficios recaudados a consecuencia del uso de la red se invirtieron en mejorar la calidad de Internet.

Esta somera aproximación histórica permite dar una definición de Internet como un conjunto de millares de redes de ordenadores interconectadas a través de todo el mundo. Cuando dos ordenadores se conectan en Internet siempre hay uno que trabaja, llamado el servidor, y otro que recibe el producto del trabajo de éste y que se denomina cliente. En la terminología informática se diría que se utiliza una arquitectura cliente-servidor. El proceso de comunicación entre ambos se realiza de la siguiente manera: el ordenador cliente llama a la puerta principal del ordenador servidor para pedirle una información concreta. El ordenador servidor, que se encuentra siempre escuchando cualquier alteración de la red que le llegue por esa puerta principal, no responde directamente sino que genera un proceso hijo que se encarga de negociar con el cliente por qué otra puerta será atendida su petición ${ }^{6}$. De esa forma la puerta principal queda libre casi siempre, lo que permite que se multiplique el número de clientes que pueden acceder al servidor sin que se formen interminables colas ${ }^{7}$. Las demoras en la comunicación suelen deberse a la limitación de las líneas de comunicación ${ }^{8}$.

\footnotetext{
Cuando las negociaciones entre el proceso hijo y el cliente no finalizan, los informáticos suelen hablar de un proceso zombie.

7 A pesar de la rapidez con que se actúa hay servidores que motu propio limitan el número de clientes que pueden acceder a su servicio.

s La Telefónica española ha conseguido uno de los mayores índices de demora.
} 
Pero, ¿para qué sirve Internet? o ¿qué servicios da Internet a los que la utilizan? En la actualidad Internet ofrece un gran número de servicios a los abonados entre los que hay que señalar el correo electrónico, los grupos de interés, y el World Wide Web ${ }^{9}$. Personalmente creo que estos tres servicios pueden $y$ deben ser utilizados para la enseñanza a distancia $y$, de una forma especial, por un profesor de Historia Contemporánea de la Universidad Nacional de Educación a Distancia. Al mismo tiempo que se exponen ejemplos de estas aplicaciones parece oportuno explicar, con algo más de detenimiento, estos tres servicios fundamentales que ofrece la red Internet.

\section{CORREO ELECTRÓNICO}

El correo electrónico (e-mail), posiblemente el servicio más conocido de Internet, permite enviar mensajes $\mathrm{y}$, por tanto, información a cualquier miembro de la red a nivel mundial. Como cualquier técnica de comunicación, el correo electrónico tiene sus ventajas e inconvenientes. La velocidad de transmisión es algo menor que la del teléfono, pero mucho mayor que la del correo, sobre todo en determinados países como el nuestro, de tal forma que el mensaje llega en un brevísimo espacio de tiempo a cualquier parte del mundo. Al igual que el correo tradicional, el correo electrónico es también asincrónico; el emisor envía el mensaje cuando lo considera necesario y el receptor lo lee cuando desea lo que supone una cierta comodidad sobre todo cuando se trata de establecer comunicación con alguien que se halla a una gran distancia o cuando no se encuentra disponible porque los horarios son muy distintos. Otra ventaja clara estriba en que no existe una posible dualidad de direcciones y, por tanto, es imposible que el mensaje se pierda. Si se conservan las copias de seguridad, los mensajes enviados y recibidos pueden ser recuperados tal como ocurrió con los de Oliver North en el Irangate. Finalmente el correo electrónico es más económico que una carta o un fax y, desde luego, más que una llamada internacional.

Para poder usar el correo electrónico es necesario poseer una dirección y utilizar el software apropiado. A primera vista la dirección electrónica,

9 Una explicación detallada y clara de todos los servicios que ofrece Internet se encueritra en el folleto Guide to Network Resource Tools publicado por la Red europea académica y de investigación (European Academic \& Research Network). La versión consultada, la 3.0, es de mayo de 1994 y se puede conseguir con un mensaje electronico a listserv@earncc.ear.net utilizando la orden get nettools.txt. 
por ejemplo avelasco@sr.uned.es, parece un oscuro y confuso galimatías; se entiende algo, pero no todo. Como puede verse, consta de dos partes divididas por el signo de la arroba, @: la primera es una abreviatura del nombre personal del que utiliza el correo electrónico, mientras que la segunda indica el ordenador central que está conectado a la red Internet ${ }^{10}$. En el ejemplo anterior sería el ordenador situado en el edificio de la calle Senda del Rey (sr) de la Universidad Nacional de Educación a Distancia (uned) de España (es). En los Estados Unidos, porque fueron los pioneros, no hay indicativo del pais pero sí del ámbito o materia sobre la que trabaja el ordenador ${ }^{11}$.

En cuanto al software, existe un sinnúmero de programas de correo electrónico para cada tipo de ordenador y para cualquier sistema operativo ${ }^{12}$. En el entorno Window los más conocidos son Eudora y Pegasus ${ }^{13}$. La Universidad Nacional de Educación a Distancia utiliza el segundo de ellos, por lo que será éste el que sirva de apoyo para explicar o mostrar el funcionamiento del correo electrónico, cuya pantalla principal es la siguiente.

La estructura de un mensaje en el correo electrónico se ha estandarizado para que no exista confusión y está constituida básicamente por cuatro partes claramente diferenciadas: la dirección electrónica del destinatario a quien se envía el mensaje (To:), el tema o resumen del mensaje (Subj:), el contenido del mensaje (para lo que existe un espacio en blanco donde se se puede escribir lo que se quiera) y la dirección electrónica del remitente que normalmente no hay que escribirla porque se presupone

10 En realidad, la dirección son números; concretamente cuatro grupos de ocho bits, de forma que cada grupo puede alcanzar valores entre $O$ y 255. Estos cuatro grupos se combinan en una notación llamada dotted quad en la que se distinguen las distintas secciones gracias ai punto que las divide. Como es lógico es más difícil recordar130.206.52.2 que avelasco@sr.uned.es.

1 Los principales ámbitos o dominios de jerarquia superior son los siguientes:

\begin{tabular}{|c|l|}
\hline DOMINIO & \multicolumn{1}{|c|}{ ÁMBITO } \\
\hline com & Empresas comerciales \\
edu & Instituciones educativas \\
gov & Dependencias gubernamentales \\
mil & Organizaciones militares \\
org & Otras organizaciones \\
net & Recursos de la red \\
\hline
\end{tabular}

12 Como en todo caso de transferencia de información estos programas utilizan un protocolo común, llamado SMTP (Simple Mail Transfer Protocol) que forma parte del conjunto que ofrece TCP/IP.

13 Estos programas se encuentran en Software Callection 0.1, CD-ROM publicado por el Departamento de Filología Germánica de la UNED. 
que es la del usuario como se indica en la barra inferior del programa. El programa permite con carácter opcional el acuse de recibo, el modo urgente, la confirmación de la lectura o el cifrado del mensaje ${ }^{14}$.

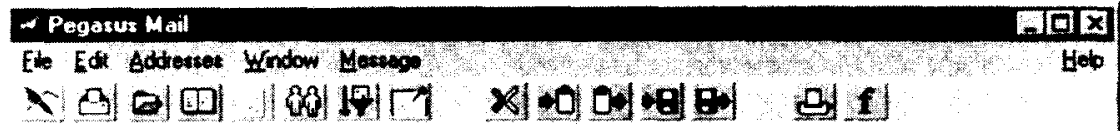

Desde el punto de vista docente el correo electrónico es un medio utilisimo para mantener una fluida comunicación con los alumnos a nivel individual. Ahora bien, no se trata sólo de poder sustituir la carta o la conversación telefónica, sino de poder enviar a través de la red material informático o previamente informatizado ${ }^{15}$. Por ejemplo, a aquellos alumnos

${ }^{14}$ Al contrario de lo que sucede con el correo normal y las llamadas telefónicas que son bastante seguras, (salvo excepciones como las ocurridas con el CESID), no existe en internet limitación alguna contra las lecturas de los mensajes, lo que obliga a codificarlos si se requiere un alto grado de confidencialidad. Los sistemas más típicos para encriptar son el ROT13 y el DES que naturalmente no previenen de una intencionada decodificación pero por lo menos evitan la lectura accidental.

15 Cuando se envía un mensaje a través de Internet las letras o simbolos se convierten en números comprendidos entre el O y el 127, es decir, se utiliza el código ASCII (American Standard Code for Information interchange). Los ficheros que están compuestos por un código binario puro se puẹden convertir en una representación que esté localizada también entre el $O$ y el 127 tomando de esta forma la estructura de un simple mensaje aunque realmente no lo sea. El sistema de conversión se conoce por UUENCODE y, naturalmente, al recibirse debe restituirsele la forma original con ayuda de UUDECODE. Todos estos pasos se hacen de forma automática. 


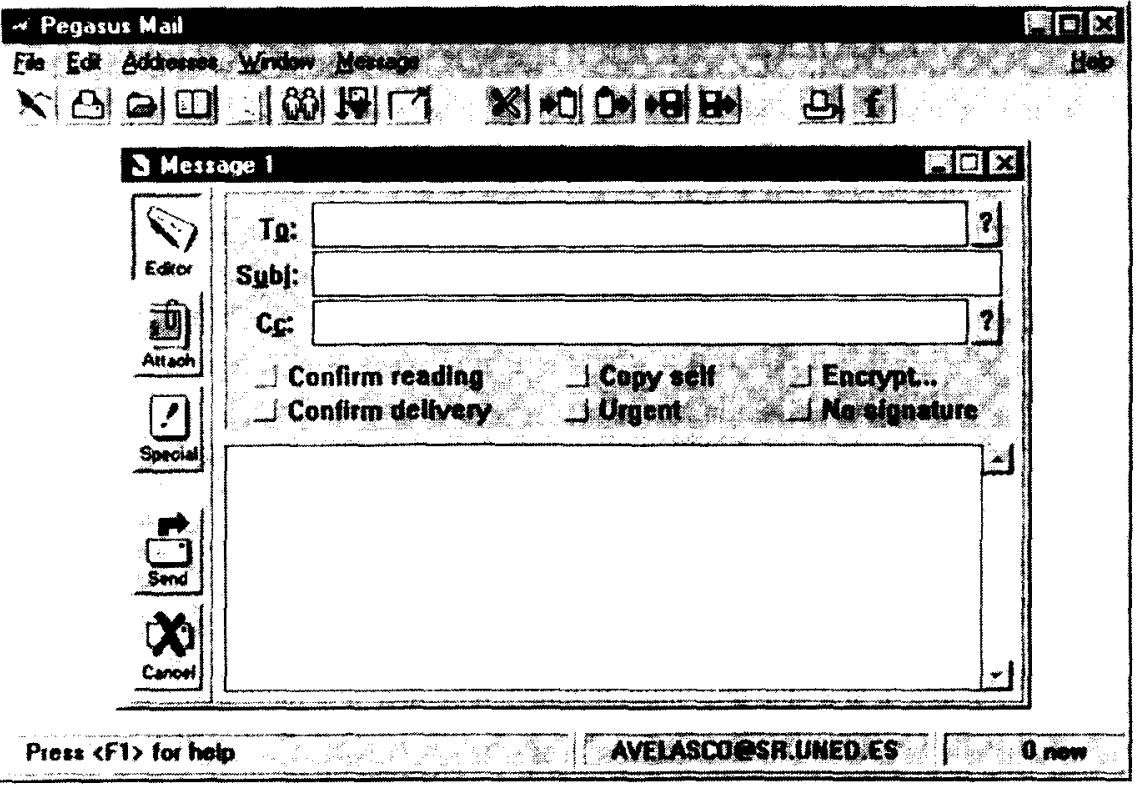

que han comenzado la elaboración de la tesis doctoral se les puede remitir, unido a un mensaje normal, un programa sencillo de bibliografía como el Biblio.exe o una lista bibliográfica originada por esta última aplicación. La operación es sencilla utilizando la función de "Añadir documento" representada por el icono Attach:

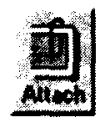

Ahora bien, la utilidad del correo electrónico no se reduce a la comunicación personal entre dos interlocutores, sino que también se puede utilizar para enviar el mismo mensaje a varios destinatarios, tal como ocurre cuando se manda una circular a todos los tutores del departamento. En el caso de la asignatura "Historia Contemporánea de España", que por encargo del departamento codirijo, se ahorraría como mínimo el tiempo que se tarda en hacer cuarenta y una fotocopias, introducirlas en sus respectivos sobres, hacer las etiquetas de las direcciones, pegarlas y, finalmente, enviarlas al servicio de correos de la UNED para que al cabo de veinticuatro horas empezasen a circular lentamente por las estrechas y tortuosas veredas postales. 


\section{LOS GRUPOS DE INTERÉS}

El segundo servicio que ofrece Internet y que puede y debe ser utilizado por un profesor de Historia Contemporánea de la Universidad Nacional de Educación a Distancia son los grupos de interés. En ellos hay que distinguir entre USENET y las listas de distribución. USENET, que es un servicio y no una red como a menudo aparece en las revistas españolas de divulgación informática, tiene como misión principal organizar una distribución rápida y eficiente en una serie de áreas de todo un conjunto de noticias y comunicaciones electrónicas que circulan por toda la red Internet. Para un gran número de personas USENET es el corazón de la red y, desde luego, el servicio que dispone de una más amplia difusión y una mayor popularidad probablemente debido al estrecho nexo humano que lleva consigo. Hay una enorme muchedumbre de personas ávidas de noticias y con necesidad de comunicarse que en pleno y continuo movimiento reciben y emiten información a través de USENET. El envío y recepción de estos mensajes a estas áreas puede servir como punto de partida para el conocimiento personal, el intercambio de ideas, etc., entre distintas personas.

Estas áreas de información electrónica giran en torno a un tema concreto al que se adhiere un grupo de usuarios de Internet. Naturalmente hay una gran cantidad de áreas que se refieren a todas las materias que tienen relación con la informática, pero la variedad de temas discutidos es enorme y crece continuamente. Al conjunto de personas que intercambian información sobre un tema concreto en un área se les denomina grupos de interés (Newsgroups), foros de discusión o grupos de debate ${ }^{16}$. Se calcula que actualmente hay cerca de diez mil grupos de discusión en USENET y que sus discusiones generan más de siete millones de palabras escritas cada día. En los foros de discusión se expone un mensaje de forma que los usuarios interesados puedan leerlo libremente e incluso responder. Cada foro es como un gran tablón de anuncios en el que los lectores "pinchan» sus mensajes y las contestaciones a los mismos creando de hecho varios hilos de conversación según los subtemas que vayan saliendo.

Debido a la gran masificación de foros, fue necesario organizar un sistema de identificación que asignase un nombre a cada foro. La solución consistió en dividir el nombre en una serie de niveles identificativos. El

16 La palabra castiza que más se aproxima a la idea es la "tertulia" y, si se les priva de su aspecto político, las actuales tertutias radiofónicas. 
foro de discusión sobre la Segunda Guerra Mundial, por ejemplo, tiene como nombre soc. history. war.world-war-ii. El primer nivel, soc, es un identificador genérico que permite saber si el foro es de carácter técnico, social, recreativo, científico, etc ${ }^{17}$. El siguiente nivel, history, informa del tema principal del foro, mientras que war indica el tema secundario. Un cuarto nivel, world-war-ii, se suele introducir en el caso de recibir un elevado volumen de mensajes diariamente de forma que el filtrado de los diferentes temas se realice automáticamente. En algunos casos donde la complejidad de los temas puede sembrar la confusión en el momento de enviar un mensaje, puede incluso llegarse a ver seis niveles distintos.

Todo el proceso de envío y recepción de mensajes es llevado a cabo por uno de los numerosos programas especializados en este tipo de tareas. Los dos programas de gestión de USENET más frecuentemente utilizados en el entorno Windows son Paperboy y Winvn, y su facilidad de manejo es reconocida por todos. No existe limitación escrita alguna respecto al comportamiento dentro de USENET, aunque por regla general se siguen una serie de directrices llamadas Nettiquette ${ }^{18}$. La gestión de USENET no la lleva a cabo ninguna persona en concreto sino que más bien es moderada por toda la comunidad suscrita al foro; sin embargo, a veces hay algunos que disponen de usuarios voluntarios llamados moderadores cuya misión es filtrar los mensajes que, tanto por la forma como por el fondo, no son apropiados al tema principal del debate. Tal ocurre en el grupo dedicado a la Segunda Guerra Mundial, soc.history.war.world-war-ii, donde los moderadores se niegan a aceptar los artículos revisionistas que proponen la inexistencia del holocausto ${ }^{19}$.

7 Estos son algunos de los identificadores más conocidos:

\begin{tabular}{|c|l|}
\hline IDENTIFICADOR & CATEGORIA \\
\hline biz & Negocios \\
comp & Ordenadores \\
news & Noticias generales \\
rec & Recreativos \\
sci & Cientifico \\
soc & Social \\
talk & Orientado al debate \\
misc & Miscelánea \\
\hline
\end{tabular}

18 Estas normas de etiqueta se centran fundamentalmente en una gran delicadeza en el trato, de tal forma que no es raro encontrarse con duras contestaciones de muchos participantes por parecer que se ha sido ofensivo con algún miembro del toro.

${ }_{19}$ Las reglas principales que siguen los moderadores del grupo dedicado a la Segunda Guerra Mundial son las siguientes: " Articles which discuss World War Two and its events will be approved for posting.- * Articles arguing that the Holocaust never happened will not be approved 
Los grupos de discusión proporcionan una valiosa información desde el punto de vista docente puesto que permiten localizar lugares, sitios dicen los informáticos, donde pueden encontrarse libros, artículos, gráficos, mapas, bibliografía o cronologías que pueden servir de apoyo para la enseñanza de la historia. Gracias a las indicaciones del grupo de discusión soc.history.war.world-war-ii se pueden conseguir la Carta Atlántica firmada por Roosevelt y Churchill el 14 de agosto de 1944 y los documentos de la capitulación sin condiciones de los ejércitos alemanes en toda Europa en mayo de $1945^{20}$. Indudablemente esta documentación es de interés para cualquier alumno de Historia contemporánea.

El segundo componente del servicio "grupos de interés» que ofrece Internet es el de las listas de distribución (mailing list). En una primera aproximación se podría describir una lista de distribución como una dirección de correo electrónico que contiene un conjunto de direcciones de personas que han mostrado claramente su interés por un tema común. A primera vista podría decirse que las listas de distribución son una tautología de USENET. Sin embargo, hay varias diferencias substanciales entre ambos. La primera de ellas radica en su accesibilidad. Mientras en USENET el acceso es totalmente abierto, en las listas de distribución se restringe exclusivamente a aquellas personas que han decidido fehacientemente formar parte de ellas. Si para comprender los grupos de USENET sirve la imagen de un tablón público de anuncios donde cualquiera puede poner un papel para que sea leído por cualquier transeúnte, las listas de distribución se podrían comparar a las tertulias decimonónicas en los casinos donde se ejercía al máximo la reserva del derecho de admisión. Esta restricción en el acceso es lo que ha llevado a que las listas de distribución tengan un carácter más serio, menos popular, que los grupos de USENET ${ }^{21}$. A esta diferencia hay que añadir que el mensaje o información que se sitúa en una lista de distribución llega a cada uno de los miembros de la lista de tal manera que todo lo que uno

for posting.- * Articles must contain original thought. Lengthy quoting of source material with a couple of lines of comment at the end will not be approved for posting.- * Blank messages, test messages, advertisements, MAKE.MONEY.FAST, and so forth, will not be approved for posting.* Articles which include excessive quoting (e.g. an article which quotes an entire other article in order to add a few comments at the end) will be trimmed down by the moderator in question.- *An article MUST have a valid reply-to address or it will not be approved for posting.- * Rejected articles will be shared with the other moderators for group consideration". FAQ de soc.history.war.world-war-il.

${ }^{20}$ La Carta Atlántica se halla en el archivo charter.txt y los documentos en german-s.txt. Originariamente se encuentran en el ordenador central de la Universidad de Missisipi: www.msstate.edu/Archives/History/USAM/WII/charter.txt y www.msstate.edu/Archives/History/USA/WWII/ german-surrender.

${ }_{21}$ Lo cual no es óbice para que existan listas de distribución de astrología, por ejemplo. 
escribe lo leen los demás o, al menos, lo pueden leer. La última diferencia básica estriba en el uso del correo electrónico, con lo que se evita el tedioso trabajo de tener que aprender un nuevo programa como ocurre en USENET con los citados Paperboy y Winvn.

El número de listas de distribución es muy elevado pero fácilmente conseguibles porque solamente hay que enviar una solicitud, naturalmente por correo electrónico, a un ordenador que tenga o pueda conseguir todas las listas de distribución. Se calcula que actualmente hay más de 250 ordenadores en más de treinta países que tienen todas las listas de distribución conocidas ${ }^{22}$. Para conseguir un listado de todas las listas de distribución con una breve explicación de su contenido sólo hay que enviar el

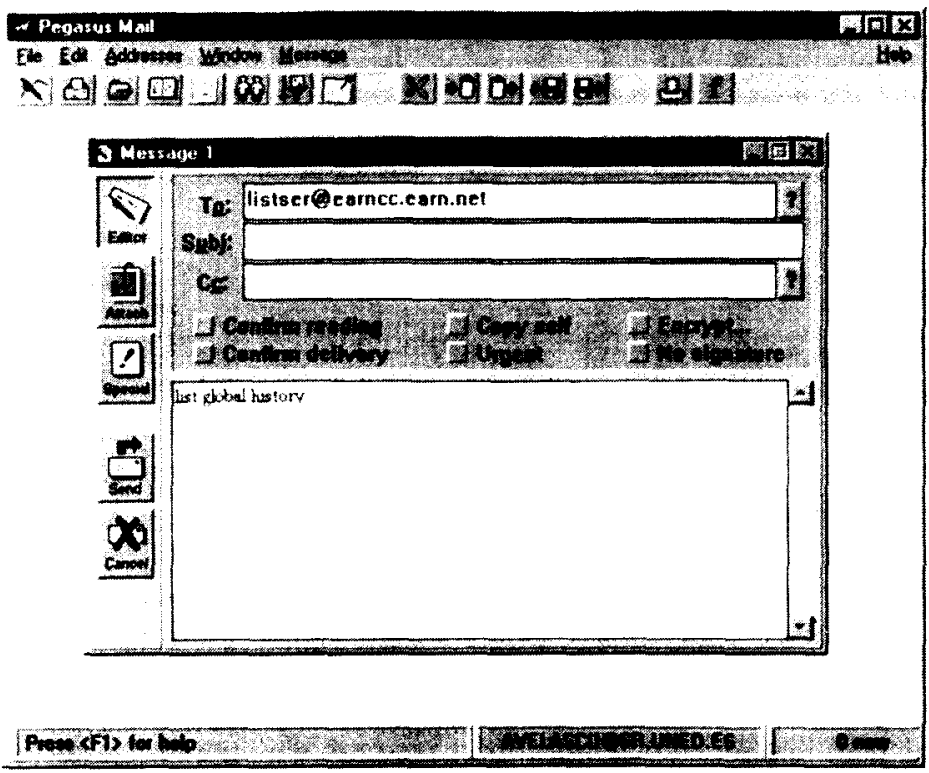

22 Los principales ordenadores que poseen estas listas son:

\begin{tabular}{|l|l|l|}
\hline ORDENADOR & \multicolumn{1}{|c|}{ INSTITUCIÓN } & \multicolumn{1}{|c|}{ PAIS } \\
\hline EARNCC & EARN & Francia \\
DEARN & GMD & Alemania \\
HEARN & Universidad Católica de Nimega & Holanda \\
SEARN & Real Escuela Técnica Superior de Estocolmo & Suecia \\
BITNIC & Centro de Información de BITNET & Estados Unidos \\
PUCC & Universidad de Princenton & Estados Unidos \\
\hline
\end{tabular}


mensaje mostrado en la figura siguiente a cualquiera de los anteriores ordenadores, aunque conviene hacerlo a uno que esté próximo, como el EARNCC de la ya citada Red europea académica y de investigación (European Academic \& Research Network).

En puridad se ha enviado una orden, el comando list global history, al ordenador EARNCC cuya dirección electrónica es listserv@list.earn.net. El resultado, obtenido en cuestión de minutos, es el conjunto de listas de distribución que en la explicación de su contenido contengan la palabra history y que se muestra a continuación

$\begin{array}{ll}\text { AERA-F } & \text { AERA-F@ASUVM.INRE.ASU.EDU } \\ & \text { AERA-F Division F: History and Historiography }\end{array}$

AEROSP-L AEROSP-L@SIVM.SI.EDU

Aeronautics \& Aerospace History

AMERCATH AMERCATH@UKCC.UKY.EDU AMERCATH - A DISCUSSION LIST ON THE HISTORY OF AMERICAN CATHOL+

AMWEST-H AMWEST-H@UMRVMB.UMR.EDU

AmWest-H - American West History Forum

ANCIEN-L ANCIEN-L@ULKYVM.LOUISVILLE.EDU

History of the Ancient Mediterranean

ASTR-L $\quad$ ASTR-L@VMD.CSO.UIUC.EDU

Theatre History Discussion List - Amer. Soc. for

Theatre Reseat

ATHG ATHG@MIAMIU.MUOHIO.EDU

Alcohol and Temperance History Group

AZTLAN AZTLAN@ULKYVM.LOUISVILLE.EDU

Pre-Columbian History

CHPSSTU CHPSSTU@UMDD.BITNET

Committee On The History And Philosophy Of Science At UMCP Stu+

COMHIST COMHIST@VM.ITS.RPI.EDU

History of human communication

E-CANADA E-CANADA@UICVM.UIC. EDU

History of Canada Editorial Board List

EARLYSCIENCE-L EARLYSCI ENCE-L@LISTSERV.VT. EDU History of Science Society - Early Science Interest Group 
ELIAS-I ELIAS-I@NIC.SURFNET.NL

Figurational Studies in Social Science, History and Psychology

EMHIST-L EMHIST-L@RUTVM1.RUTGERS.EDU EMHIST-L Early Modern History Forum

ESPORA-L ESPORA-L@UKANVM.CC.UKANS.EDU History of the Iberian Peninsula

ETHNOHIS ETHNOHIS@NIC.SURFNET.NL General Ethnology and History Discussion List

GAHIST-L GAHIST-L@UGA.CC.UGA.EDU Georgia History Discussion List

H-AFRICA H-AFRICA@MSU.EDU

H-NET List for African History

H-ALBION H-ALBION@MSU.EDU

H-Net British and Irish History List

H-ALBION@UICVM.UIC.EDU

H-NET British and Irish History List

H-AMREL H-AMREL@MSU.EDU

$\mathrm{H}$-Net American Religious History discussion group

H-ANTIS H-ANTIS@UICVM.UIC.EDU

History of Antisemitism List

H-ASIA H-ASIA@MSU.EDU

$\mathrm{H}$-Net list for Asian History and Culture

H-ASIA@UICVM.UIC.EDU

$\mathrm{H}$-Net list for Asian History

H-CANADA H-CANADA@MSU.EDU

$\mathrm{H}$-Net List for Canadian History

H-CIVWAR H-CIVWAR@MSU.EDU

$\mathrm{H}$-Net US Civil War History discussion list

H-CIVWAR@UICVM.UIC.EDU

H-Net US Civil War History discussion list

H-DIPLO H-DIPLO@MSU.EDU

H-Net Diplomatic History List

H-DIPLO@UICVM.UIC.EDU

$\mathrm{H}$-Net Diplomatic History list

H-ETHNIC H-ETHNIC@MSU.EDU

H-NET List on Ethnic History 
H-ETHNIC@UICVM.UIC.EDU

$\mathrm{H}$-Net Ethnic History discussion list

H-FILM H-FILM@UICVM.UIC.EDU

An H-Net List for Film History and Studies

H-FRANCE H-FRANCE@UICVM.UIC.EDU

$\mathrm{H}$-Net History of France List

H-FRANCE@VM.CC.PURDUE.EDU

H-NET French History discussion group

H-GERMAN H-GERMAN@MSU.EDU

H-NET List on German History

H-GERMAN@UICVM.UIC. EDU

German History list

H-GRAD H-GRAD@UICVM.UIC.EDU

$\mathrm{H}$-Net History Graduate Students discussion list

H-IDEAS H-IDEAS@UICVM.UIC.EDU

H-NET Intellectual History List

H-ITALY H-ITALY@UICVM.UIC.EDU

Italian History List from $\mathrm{H}-\mathrm{NET}$

H-LABOR H-LABOR@MSU. EDU

$H$-Net Labor History discussion list

H-LABOR@UICVM.UIC.EDU

$\mathrm{H}$-Net Labor History discussion list

H-LATAM H-LATAM@MSU.EDU

H-Net Latin-American History List

H-LATAM@UICVM.UIC.EDU

$\mathrm{H}$-Net Latin American History discussion list

H-LAW H-LAW@MSU.EDU

$\mathrm{H}$-Net and ASLH Legal History Discussion list H-LAW@UICVM.UIC. EDU

$\mathrm{H}$-Net and ASLH Legal History Discussion list

H-LOCAL H-LOCAL@MSU.EDU

H-Net DISCUSSION LIST FOR LOCAL AND STATE HISTORY

H-MAC H-MAC@MSU.EDU

H-NET list for the History and Macintosh Society

H-POL H-POL@KSUVM. KSU. EDU

$\mathrm{H}$-Net Political History discussion list 
H-POL@UICVM.UIC.EDU

$\mathrm{H}-\mathrm{Net}$ Political History discussion list

H-RHETOR H-RHETOR@MSU.EDU

$\mathrm{H}-\mathrm{Net}$ History of Rhetoric Discussion list

H-RHETOR@UICVM.UIC. EDU

H-NET HISTORY OF RHETORIC DISCUSSION LIST

H-RURAL H-RURAL@MSU.EDU

An H-Net list for discussion of Rural \& Agricultural History H-RURAL@UICVM.UIC.EDU

An H-NET list for discussion of Rural \& Agricultural History

H-RUSSIA H-RUSSIA@MSU.EDU

H-Net Russian History list

H-RUSSIA@UICVM. UIC. EDU

$\mathrm{H}$-Net Russian History list

H-SOUTH H-SOUTH@MSU .EDU

$\mathrm{H}$-Net Southern History Discussion List

H-TEACH H-TEACH@MSU.EDU

H-Net List for Teaching College History and Related

Fields

H-TEACH@UICVM. UIC.EDU

$\mathrm{H}-\mathrm{Net}$ List for Teaching History and Related Fields

H-URBAN H-URBAN@UICVM.UIC.EDU

$\mathrm{H}$-Net Urban History discussion list

H-WAR H-WAR@KSUVM. KSU.EDU

H-Net Military History Discussion List

H-WEST H-WEST@MSU.EDU

H-Net Western History List

H-WEST@UICVM.UIC.EDU

$\mathrm{H}$-Net Western History List

H-WOMEN H-WOMEN@MSU.EDU

H-NET List for Women's History

H-WOMEN@UICVM.UIC.EDU

$\mathrm{H}$-WOMEN Women's History discussion list

H-WORLD H-WORLD@MSU.EDU

H-NET List for World History

HASTRO-L HASTRO-L@WVNVM.WVNET.EDU

History of Astronomy Discussion Group 


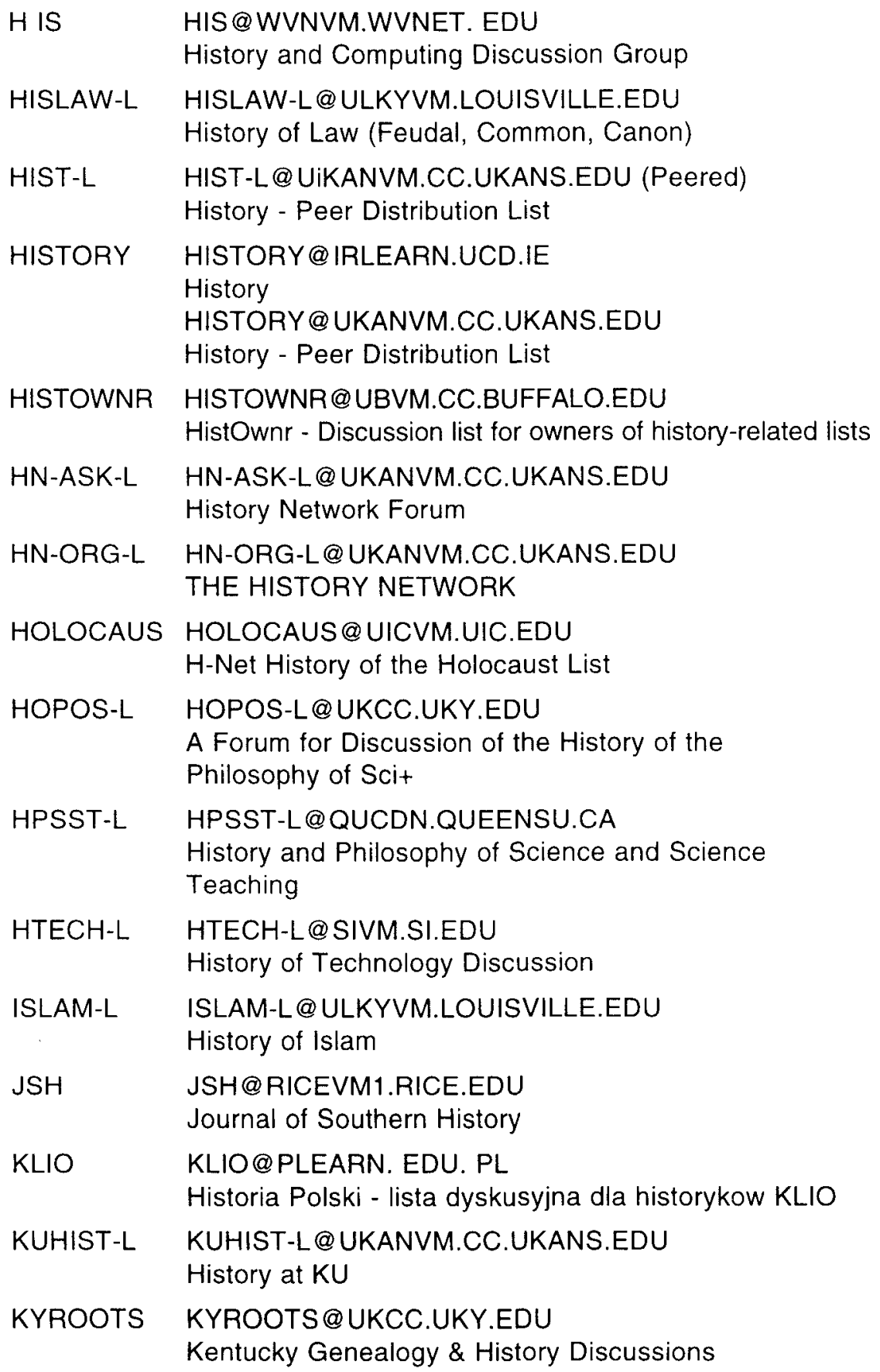




\begin{tabular}{|c|c|}
\hline MAPHIST & $\begin{array}{l}\text { MAPHIST @ HARVARDA.BITNET } \\
\text { Map History Discussion List }\end{array}$ \\
\hline MEDART-L & $\begin{array}{l}\text { MEDART-L@UTORONTO.BITNET } \\
\text { MEDART-L - Medieval Art History }\end{array}$ \\
\hline MEDIEV-L & $\begin{array}{l}\text { MEDIEV-L@UKANVM.CC.UKANS.EDU } \\
\text { Medieval History }\end{array}$ \\
\hline MENA-H & $\begin{array}{l}\text { MENA-H@ULKYVM.LOUISVILLE.EDU } \\
\text { History of the Mideast and North Africa }\end{array}$ \\
\hline MHLHST-L & $\begin{array}{l}\text { MILHST-L@UKANVM.CC.UKANS.EDU } \\
\text { Military History }\end{array}$ \\
\hline MISSIONS & $\begin{array}{l}\text { MISSIONS@YALEVM.CIS.YALE.EDU } \\
\text { Missions History Discussion Group (MISSIONS) }\end{array}$ \\
\hline OHA-L & $\begin{array}{l}\text { OHA-L@UKCC.UKY.EDU } \\
\text { Oral History Association Discussion List }\end{array}$ \\
\hline PERSIA-L & $\begin{array}{l}\text { PERSIA-L@EMUVM1.CC.EMORY.EDU } \\
\text { Jewish Literature and History in the Persian Period }\end{array}$ \\
\hline RENAIS-L & $\begin{array}{l}\text { RENAIS-L@ULKYVM.LOUISVILLE.EDU } \\
\text { Early Modern History - Renaissance }\end{array}$ \\
\hline RUSHIST & $\begin{array}{l}\text { RUSHIST@EARN.CVUT.CZ (Peered) } \\
\text { RusHist- Russian History Forum } \\
\text { RUSHIST@UMRVMB. UMR. EDU (Peered) } \\
\text { RusHist- Russian History Forum }\end{array}$ \\
\hline SHARP-L & $\begin{array}{l}\text { SHARP-L@IUBVM.UCS.INDIANA.EDU } \\
\text { SHARP-L Society for the History of Authorship, Reading } \\
\text { \& Publi+ }\end{array}$ \\
\hline
\end{tabular}
\& Publit

SHOTHC-L SHOTHC-L@SIVM.SI.EDU History of Computing Issues

SISTER-L SISTER-L@LISTSERV.SYR.EDU History \& contemporary concerns of Catholic women religious

SLAVERY SLAVERY@UHUPVM1.UH.EDU

The history of slavery, the slave trade, abolition, and emancipat+

SOVHIST SOVHIST@EARN.CVUT.CZ (Peered)

SovHist - Soviet History Forum

SOVHIST@UMRVMB.UMR.EDU (Peered)

SovHist - Soviet History Forum 


\section{SPORTHIST SPORTHIST@PDOMAIN.UWINDSOR.CA ISPHES - Sport History Scholars List}

\section{STUDIUM STUDIUM@CC1.KULEUVEN.AC.BE} University history discussion list

$\begin{array}{ll}\text { TAMHA } & \text { TAMHA@CMS.CC.WAYNE.EDU } \\ & \text { Teaching American History } \\ \text { WHIRL } & \text { WHIRL@PSUVM.PSU.EDU } \\ & \text { Women's History in Rhetoric and Language } \\ \text { WHR-L } & \text { WHR-L@PSUVM.PSU.EDU } \\ & \text { Women's History in Rhetoric }\end{array}$

WORLD-L WORLD-L@UBVM.CC.BUFFALO.EDU World-L - Forum on non-Eurocentric world history

De una ligera observación de la lista precedente se pueden deducir varias conclusiones. La primera de ellas es la utilidad que puede tener, al menos teóricamente, para los historiadores cuya especialización se ve reflejada en alguna de las listas de distribución; tal sería el caso de las que tratan sobre historia diplomática como h-diplo@msu.edu. Para algunas disciplinas científicas, segunda conclusión, es muy fácil encontrar las listas de distribución de sus propias materias, pero no ocurre lo mismo con la ciencia histórica puesto que, al ser interdisciplinar, puede haber temas que interesan y que se localizan en otros campos como es el caso de la demografía. $Y$, como última conclusión, hay que hacer notar la pobreza con que aparece la Historia de España en estas listas donde sólo es tratada, en julio de 1995, en una única lista de distribución, la de la Universidad de Kansas, espora-l@ukanvm.cc.ukans.edu,compartiendo el espacio electrónico con Portugal. Esta pobreza raya con la más absoluta miseria si se piensa en la Historia Contemporánea de nuestro país.

La explicación que se da junto con el nombre de la lista es a todas luces escasa, pero para paliar este inconveniente hay artículos electrónicos que suelen ampliar el contenido de las mismas. Tal ocurre, por ejemplo, con el trabajo Electronic Sources for West European History and Culture, escrito por Erwin K. Welsch, bibliotecario de la universidad de Wisconsin, donde se aclara que la lista ESPORA-L contiene bibliografías, artículos, una amplia colección de resúmenes de documentos medievales aragoneses y todas las noticias de la American Academy of Research Historians of Medieval Spain ${ }^{23}$.

23 El artículo se puede conseguir solicitándolo al autor cuya dirección electrónica es: ewelsch@vms.macc.wisc.edu 
Si se desea participar, subscribirse dicen los informáticos, en una lista de distribución es preciso indicarlo mediante un sencillo mensaje electrónico que lleve la orden SUBSCRIBE seguida del nombre de la lista tal como se muestra en la figura siguiente.

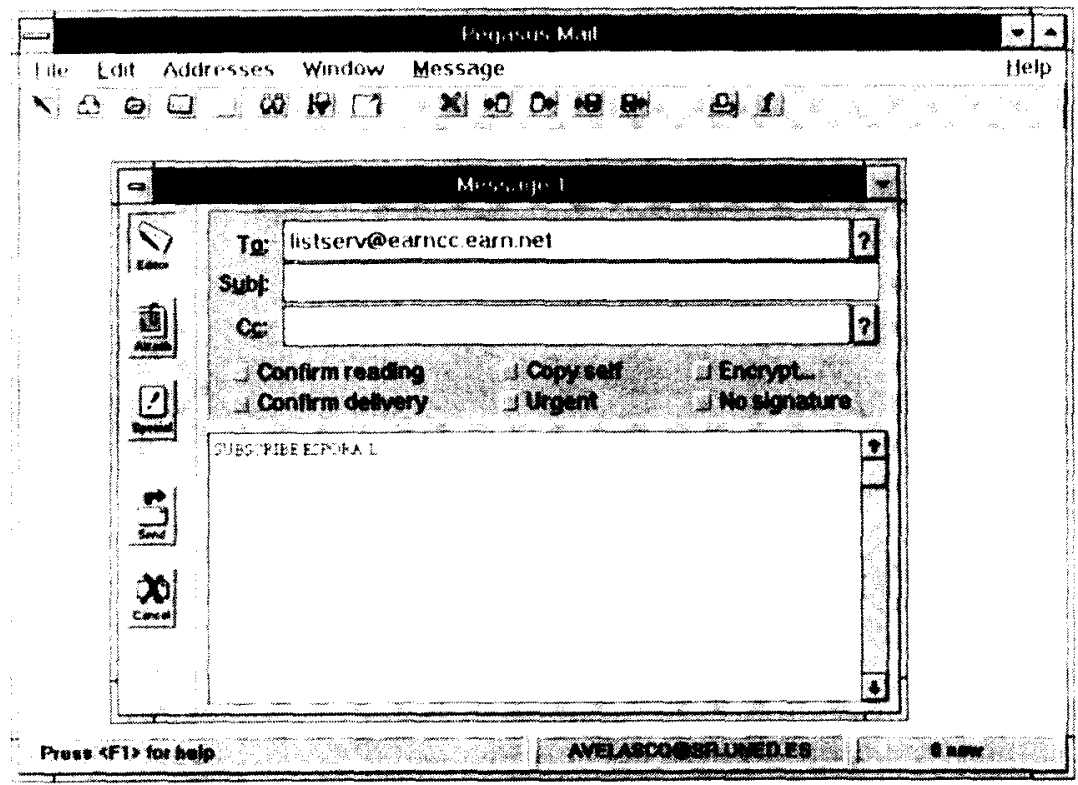

El ordenador de la EARNCC remite el mensaje recibido al ordenador que dirige la lista de distribución ESPORA-L y a los pocos minutos, cinco en este caso, se recibe un mensaje electrónico comunicando que ha sido aceptada la subscripción y que se pueden utilizar algunos comandos sencillos para retirar la suscripción, conocer las direcciones de los miembros de la lista o acusar recibo sólo a los mensajes particulares ${ }^{24}$. A partir de

24 El mensaje recibido es el siguiente:

«Date sent: Sat, 22 Jul 1995 05:26:20 -0500

From: BITNET list server at UKANVM (1.7f) <LISTSERV@UIKANVM.CC.UKANS.EDU>

Subject: Your subscription to list ESPORA-L

To: Angel Martinez de Velasco <AVELASCO@SR.UNED.ES>

Send reply to: ESPORA-L-Request@UKANVM.CC.UKANS.EDU 2

Dear networker,

Your subscription to list ESPORA-L (History of the Iberian Peninsula) has been accepted.

You may leave the list at any time by sending a "SIGNOFF ESPORA-L" Command to LIST. SERV@UKANVM BITNET (or LISTSERV@UKANVM.CC.UKANS.EDU). Please note that this com- 
ese momento siempre que cualquier miembro de la lista de distribución envie un mensaje se recibirá una copia de él.

\section{ELWWW}

La cantidad de información que almacenan los ordenadores conectados a Internet es ingente. Sería imposible la consulta de la información conservada en un archivo informático si no se supiera exactamente el nombre del mismo, el ordenador que lo contiene y la situación de este ordenador. Por ejemplo, para consultar el testimonio de Walt Disney ante el Comité de actividades antinorteamericanas el 24 de octubre de 1947, testimonio que muestra extraordinariamente el clima de crispación política existente en la postguerra norteamericana, habría que saber que el archivo que lo contiene se llama disney y que se encuentra en english.hss.cmv.edu/ OF $2 \% 3 A 2243 \%$ $3 a d i s n e y \% 20$ Testimony\%20to\%HUAC. Sería poco considerar esta tarea como obra de Titanes. Para evitar este problema existen servicios, sistemas de información es el nombre técnico, que permiten localizar y recuperar los datos que se desean de una manera eficaz y relativamente fácil. Entre todos ellos el más conocido es el World Wide Web también llamado Web, W3 o WWW.

El World Wide Web es un sistema de información basado en el hipertexto y está adquiriendo una casi total aceptación entre las personas que se conectan a Internet por su facilidad de utilización y por permitir el acceso a medios concretos de adquisición de información más técnicos y por tanto más complicados ${ }^{25}$. En sus orígenes el W3 fue desarrollado en el Laboratorio

mand must NOT be sent to the list address (ESPORA-L@UKANVM) but to the LISTSERV address (LISTSERV@UKANVM).

The amount of acknowledgement you wish to receive trom this list upon completion of a mailing operation can be changed by means of a "SET ESPORA-L option" command, where "option" may be either "ACK" (mail acknowledgement), "MSGACK" (interactive messages only) or"NOACK".

Please note that it is presently possible for anybody to determine that you are signed up to the list through the use of the "REVIEW" command, which returns the network address and name of all the subscribers. If you do not wish your name to be available to others in this fashion, just issue a "SET ESPORA-L CONCEAL" command.

More information on LISTSERV commands can be found in the LISTSERV reference card, which you can retrieve by sending an "INFO REFCARD" command to LISTSERV@ UKANVM.BITNET (or LISTSERV@UKANVM.CC.UKANS.EDU).

Virtually,

The LISTSERV management

${ }_{25}$ Es realmente sencillo emplear el WWW para comenzar una sesión de TELNET, traer un archivo por FTP o acceder a un GOPHER. Estos servicios se explican perfectamente en la ya citada Guide to Network Resource Tools. 
Europeo de Física de Partículas, CERN, pensando en crear una herramienta que sirviera para facilitar la consulta de la información acumulada en Internet. También en este caso se utiliza la arquitectura cliente-servidor mediante un programa llamado visor o examinador (browser), que une un WWW servidor con el WWW cliente ${ }^{26}$. En la Universidad Nacional de Educación a Distancia se emplea como visor el programa Netscape ${ }^{27}$ por ser el más utilizado, cuya pantalla principal se muestra en la figura siguiente.

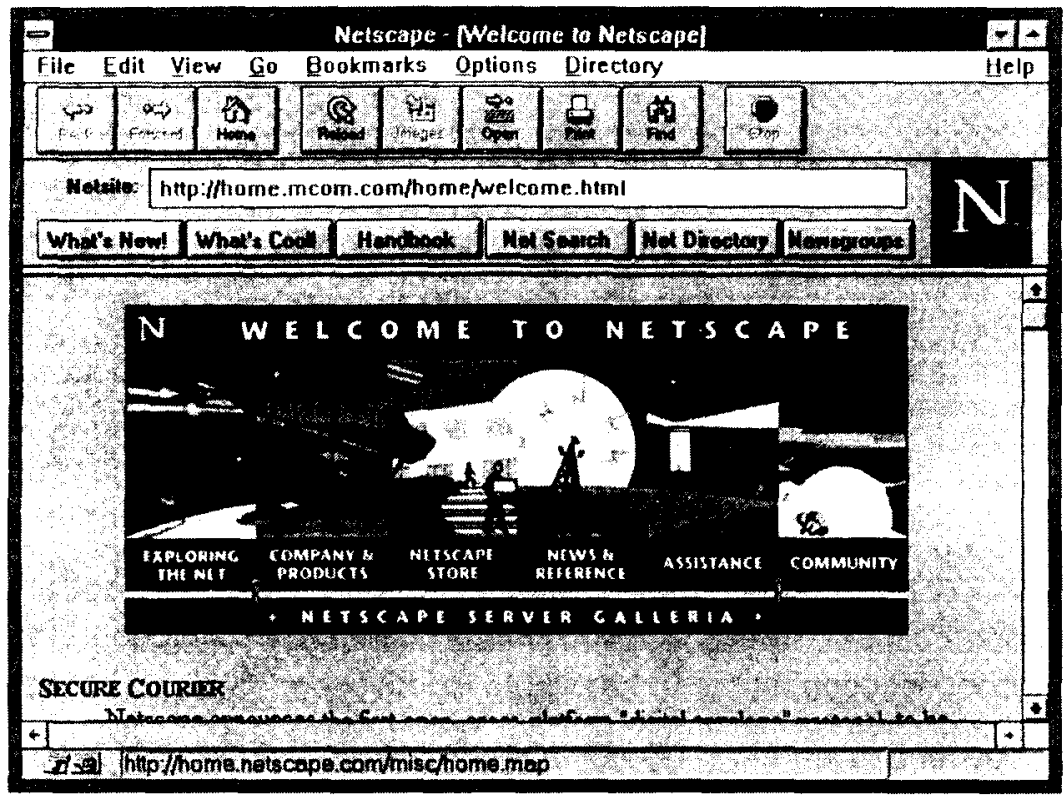

En la parte superior de la pantalla se muestran las posibilidades principales que ofrece el Netscape. Entre ellas interesa destacar que en el recuadro con el título Location o Netsite se indica perfectamente la dirección donde se encuentra el texto, página es el nombre informático, que aparece en el monitor ${ }^{28}$.

26 El visor puede estar instalado localmente, es decir en el ordenador que se está utilizando, o en una computadora remota. Si se está conectado directamente a Internet se recomienda instalar localmente el browser porque hacerlo en un ordenador remoto supone una pérdida de funcionalidad y de rendimiento.

27 Los programas más conocidos son el Netscape, Cello y Mosaic.

28 El nombre técnico, que aparece con relativa frecuencia, es URL; acrónimo de Uniform Resource Locator. 


\section{Location: http:/home.mcom.com/hometwelcome.html}

En este caso concreto se está en el archivo welcome.htm/ del subdirectorio home del ordenador home.mcom.com.y se está utilizando un protocolo de transferencia de hipertexto http (Hipertext Transfer Protocol). El recuadro de la localización permite introducir manualmente la dirección de cualquier documento que se quiera consultar, con tal de saber dónde se encuentra y qué protocolo de comunicaciones usar. ${ }^{29} \mathrm{Si}$ se quisiera que en el monitor apareciesen las citadas declaraciones de Walt Disney habría que escribir: gopher://english.hss.cmv.edu/OF-2\%3A2243\%3adisney\%20 Testimony $\% 20$ to \%HUAC. Naturalmente es bastante farragoso introducir manualmente una y otra vez todos estos elementos de localización, pero para evitar esta tarea tediosa se pueden registrar usando la posibilidad de añadir ( $\underline{A} d d$ Bookmark o $C t r l+A$ ) incluida en la opción Marca-texto (B ce de los archivos más interesantes o de las páginas del WWW que se utilizan más frecuentemente.

Dada la vasta información que contienen todos los ordenadores conectados en Internet, el programa Netscape permite buscar en aquellos sitios donde puede encontrarse un determinado tema mediante el botón de búsqueda en la red. Pulsando este botón se llega a una nueva página donde después de algo de literatura y mucho de propaganda se encuentra un recuadro para rellenar con la palabra que se desea buscar.

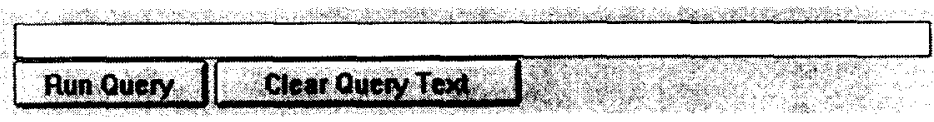

Si se escribe History of Spain es muy probable que en la pantalla del ordenador salga algo semejante a lo que muestran los dos cuadros siguientes.

29 Las abreviaturas utilizadas para los diferentes servidores son las siguientes:

\begin{tabular}{|c|l|}
\hline ABREVIATURA & SERVIDOR \\
\hline http: $/ /$ & WWW \\
ftp: $/ /$ & FTP \\
gopher: $/ /$ & GOPHER \\
news: $(\sin / /)$ & Grupos de discusión \\
teinet: $/ /$ & TELNET \\
fine: $/ /$ & Archivo de texto \\
\hline
\end{tabular}




\section{Tektronix - lideo and Networking Division}

Tektronix. check out the Profik disk recordet and other professional videg innovations

\section{Wet Search results $\quad f: y_{1}, \ldots, C, \%$}

\section{sousters}

You scarched for. hisiory of ipain

We did not lind or use the words of

To learn how to get even belier results, please see our heloful tios

L.B Owens Home Page (Relevance 547)

J. B Owens "I believe in the future because I myself participate in its construction " .. Jules Mocheiet (heck here to see Whal's New since your last nsit. Purpose. This is the home page for the Interfer teaching of $)$ B Owens of

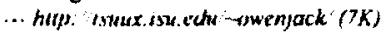

Spaill (Relevance \$38)

A chouette...(French, literally a kind of owl, but also slang fol "cool") marks expecially interesting sites General information About Spain. The CIA Factbook on Spain These are facts from 1994 This includes a small map and

-.. htip: ifflc.smu.edurlanguoges/Spann.html (6K)

Yghoo-Government Countries Spain Embassies and Copsulaces (Relevance: 537)

[ Yahoo / I p ; Search | Suggest | Add | Help] Government Coumnes Spain Embassies and Consulates

Embassy of Spain in Ottawa - exhibit on Sparush currens affairs, everyday life. history, language and culture. admin@yahoocom

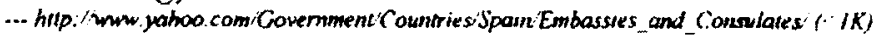

The Gumbo Shop (Relevance 536)

Flistory | Menu | Wine List | Information | Map | Recipes. Japanese | Spanish | French History Hlome , Menu | Wine List | Recipes | Information | Map On entering the pearly gates, a New Orleans Croole asked, "But Monsieur,

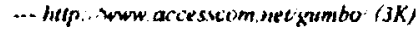

PF:RICO'S LONIF PAGE (Relevance 536)

VOTA BENE Folks. lve graduated Look for results on hntp //wuwl uniovi es My page was Ir ansplanted back in December IO when I get a commercial account this summer, look for an arnouncernent in rec spon soccer or

... hltp unw sear npermedu -arias $M A I N(J K)$

Discover Spain with the Touns1 Qffice of Sprin (Relevance 535)

Welcome to the Tourist Office of Spain Horne Page Spain is a counery nich in hision, culture and diversity - it is our hope that we can share some of this with you via the World Wide Web on the Internet Indeed. we know that you.

... hllp: inuw gol com spominadex himl (IK) 
Discover Spain with the Tourist Office of Spain (Reievance: 535)

Weicome to the Tourist Office of Spain Home Page. Spain is a country rich in history, culture and diversity - it is our hope that we can share some of this with you via the World Wide Web on the Internet Indeed, we know that you

-.- http://ww ozemail.com.aw/-spain' (IK)

ACADEMIC PROGRAMS (Relevance: 535)

Click on desired topic: . IMAGINE THE PERFECT MAJOR FOR YOU . LIBERAL STUDIES PROGRAM SAMPLE SCHEDULES SCHOOL OF ARTS AND HUMANITIES SCHOOL OF BUSINESS AND SOCIAL SCIENCES . SCHOOL OF EDUCATION AND INTEGRATED SCIENCES LASALLIAN ..

-- http://40.190.128.190/SMC/ACADPROS html (62K)

Si Spain (Relevance: 534 )

Welcome to "Si, Spain". Bienvenido a "Si, Espana". The Embassy of Spain in Ottawa (Canada) has provided this interactive service which promotes free exchange of data on Spanish current affairs and its historical and cultural development.

... http:/hwww. civeng. carleton ca/SiSpain' (3K)

Si Spain (Relevance: 534 )

Welcome to "Si, Spain". Bienvenido a "Si, Espana". The Embassy of Spain in Ottawa (Canada) has provided this interactive service which promotes free exchange of data on Spanish current affairs and its historical and cultural development.

-... http:/hww civeng.carleton.ca/SiSpain/home.html (4K)

\section{Want even more?}

InfoSeek also offers a more extensive service with access to more than 10 Web pages per search, plus lots of other interesting information--more than 10,000 Usenet newsgroups, continuous newswires, and business, computer, health and entertainment publications. Get more information about this exciting service or just sien $\mu$ for a free trial.

This free Web search service handles aver 1,000,000 queries a day and runs on an 8 CPU Sparcenter 1000 provided by Sun Microsystems

Financial support has been provided by our sponsors

Como puede observarse, la búsqueda a través de Internet ha mostrado fundamentalmente información que interesa sin lugar a dudas a los posibles turistas que visiten España. En realidad, solamente habia tres páginas de nivel universitario que pudieran interesar. En primer lugar, los programas académicos norteamericanos situados en el ordenador 140.190.128.190 que en el momento de la consulta no era accesible. En segundo lugar, una aplicación realizada en la Universidad de Oviedo que lleva el gracioso título de "Página central de Perico» y que una vez consultada no parecía ser muy académica ${ }^{30}$. Finalmente, la J.B. Owens Home Page era la

30 Todo el trabajo se refiere a las competiciones futbolísticas españolas: Calendario con resultados de la primera división de la liga de 1994-1995, plantillas de todos los equipos, historia de 
planificación de las asignaturas que imparte el Profesor J. B. Owen en la Universidad estatal de Idaho dentro de un programa de maestría sobre «Historia comparativa mundial».

De todo lo anterior se desprende la conclusión de que la Historia de España no parece estar muy bien representada en Internet aunque no ocurre lo mismo con respecto a la Historia en general. En mi opinión hay varias páginas que conviene consultar cuando se intenta observar lo que hay en Internet sobre la Historia en general: «El Servidor Inglés", los servicios de la Association for History and Computing y el índice de la Universidad de Kansas.

El Servidor Inglés, The English Server, es una de las páginas que posee más solera porque lleva ya cinco anos publicando textos de humanidades en Internet. El índice de entradas es el siguiente ${ }^{31}$ :

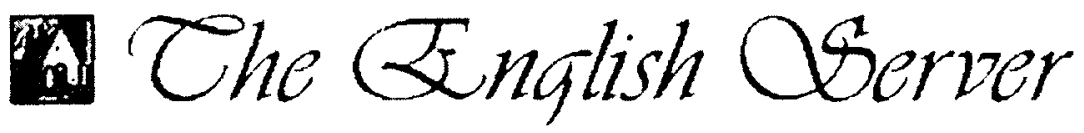

18th Century Studies

Aragonese Historiography

Artephius: The Secret Book

Attila at Chalons

Aurora of 1192

Ballots and Bullets

Baltic History

Baltic States' Independence

Bay of Pigs

Berlin Wall

Berlin. 1961-2

Canticles

Che in New York

Civil War Reading List

Collapse of USSR

Conspiracy in Sotonera

Gettysburg Address
Definitive JFK article

Dissemination of Law

Douglass: Articles

Du Bois: The Freedmen's Bureau

Eighteenth Century Studies

English Calendar

Environmentalism. Racism

Eurocentric Timeline

FDR Inaugural Address

Federalist Papers

Feudal terms

Feudalism to Socialism

Finding a Wife

Flint Sit-down

Free at Last

Garrison statement

October Revolution

la primera división con resultados (incluido el trofeo Pichichi), información sobre las competiciones de la UEFA y sobre la selección nacional española. Se incluyen imágenes de equipos y de jugadas.

31 La signatura electrónica de la página de historia de El Servidor Inglés es http://english-www. hss.cmu.edu/History.html 
Ghandi nobody knows

Haldane: Daedalus

Heritage of Stone

Hiroshima Survivors

Historical Events

History Archive at The World

History at your Fingertips

History of Morkrum Co

History of the Lib of Congress

History of the R.A.F.

History of US Labro Law

JFK Archive

Jolv: Dawn of Amateur Radio

Kansas History Archive

Laws of William

Lenin: Testament

Medieval carpentry

Medieval Pollution

Mediaeval Studies

Merck

Mississippi State History Archive

Monroe Doctrine

More: Utopia

Moscow Postcard
Online Catasto of 1427

Privatization Problems

Renaissance

Royal Society Archives

Runnion: The Negro Exodus

Russia. 1914-17

Russian Women

Russo-German relations

Smells Like Jobs

Soviet Archives

Soviet Diary

Stephens: American Thumbprints

Suicide. 1951-1988

The industrial religion

The October Surprise

Thucydides: Peloponesian War

Trotsky: Fightinz Fascism

U-2

US Historical Documents

US Inaugural Addresses

USSR in 1991

Washington: Awakeing

White: Warfare of Science

With Theology

Wood: The Capture of a Slaver

\section{Neolithic War}

Woodrow Wilson

La segunda página de WWW que merece la pena consultar es la que ofrece la Asociación para la Historia e Informática, $A H C$, titulada WWW services for Historians ${ }^{32}$. El creador de esta página ha colocado una orden en su programación para que se pueda contar automáticamente el número de usuarios que la consultan: el resultado desde el 29 de junio al 24 de julio de 1995 era de 1.006 «visitantes», lo que muestra la amplia audiencia que tiene Internet incluso en materias tan restringidas como la Historia. La información que ofrece la Association for History and Computing viene dividida, como puede verse a continuación, en varios apartados:

32 Situada en http://grid.let.rug.n/ahc/hist.htm/. La página principal con información sobre la Association for History and Computing se encuentra en http://grid.let.rug.nl/ahc/welcome.htm/. 
1. A Historian's Guide to Email and Internet Resources

2. WWW servers within the Humanities $A$ list maintained University

3. Recherche et discussions pour universitaires francophones

4. US National Endowment for the Humanities

5. The International Students of History Association (ISHA)

Calls, Requests, Activities, grants, etc.

6. Calls for papers and conference anouncements

7. Requests for help

8 Grants, fellowships, etc

Make connections with other servers

\section{Europe}

9. Netherlands Historical Data Archive (NHDA)

10. Groningen Historical Electronic Text Archive GHETA

11. SWIDOC - Amsterdam

12. Koninklijke Bibliotheek-Rubriek 15: Geschiedenis- Netherlands in Dutch

13. OnderwijsWeb - the Dutch Education Home Page

14. NSD - Norsk samfunnsvitenskapelig datatjeneste (Norway)

15. Clickable Map of Data Archives all over the world

16. Danish Data Archive

17. Heidelberg History WWW-server (German)

18. Postgraduiertenforum der Historikerlnnen der Deutschen fur Amerikastudien

19. TLTP Archeology Consortium

20. CTIH - centre for History. Archeaology \& Arthistory - Glasgow

21. The Royal Society of Edinburgh Scotland's Premier Learned Society

22. Plant Fossil Record Database

23. Institute for Historical Research - London

24. The HUMBUL Gateway - International Resources for the Humanities

25. History CD-Roms

\section{Africa}

26. AFRIA-The Africa Research \& Information Alliance

27. African Studies WebServer Australia

28. AUSTRALIA-NEW ZEALAND STUDIES CENTER, located at Penn State University

29. List of Australian Studies Centres

\section{America}

30. The Centre for Computing in the Humanities- University of Toronto

31. The Heritage Project - Canada

32. Chorus CHORUS is a WWW resource for academic and educational computing in the arts/humanities. 
33. The History Network

34. The Historical Archive of Mississippi State University

35. YAHOO

36. The Labyrinth - Medieval Studies

37. Tennessee History Gopher

38. US Holocaust Memorial Museum - Washington

39. ARTFL

40. American Studies Web

41. The Cliometric Society Server

42. Carnezie Melon History Server

43. Cornell CRP HomePage and Preserve/Net. Preserve/Net is the only comprehensive resource available for the historic preservation community on the Internet.

44. Pomona College Ancient Cultures Home Page

45. Brigham Young University - Family History

46. International Affairs Network Home Pages

47. United Nations Information Services

48. Constitutions of Countries from allover the world

49. Indiana Historical Society

50. Historical Computer Society

51. Conrad Hilton Archives and Library

52. Interdisciplinary General Education Home Page at Cal Poly. Pomona, CA

53. the historic city of Burlington, New Jersey

\section{Australia}

54. Australian Art History server

Asia

55. Yad Vashem - Israel

56. History Page of the Research Institute for the Humanities - Faculty of Arts, CUHK (Hong Kong)

57. History of Japan

\section{WWW-Historical Articles and Exhibitions}

58. History Reviews On-Line- Emagazine

59. Daniel Webster: Dartmouth's Favorite Son - A very nice looking virtual exhibition on Daniel Webster (Netscape required)

60. Hadrosaurus Foulkii - World's First Dinosaur Skeleton. Haddonfield, NJ

61. The Web Louvre - This beautiful award-winning WWW-application has one serious drawback: it is very slow.

62. WWII underground factory in Czech Republic - Have a look at a WWW-document about the factories that the Germans buitt inside mountains in the Czech Reublic during the Second World War.

63. The Commercial Computer Museum 
64. History of the Mambila of Cameroon and Nizeria - A WWW-document about the history of th Mambila tribe.

65. The Graz Arsenal - Walk with Bert Bos and George Welling through the Arsenal of Graz.

66. Euronean Historical Sites

67. WWW exhibit EXPO - This provides access to a number of WWW-expositions: a.o. 1492 at the Library of Congress, The Vatican Museum and, the Death Sea Scrols.

68. Anne Frank information - Information concerning life and death of Anna Frank and related material.

69. Greek Mythology - Everything you have always wanted to know about the relations between the classical gods, halfgods, etc.

70. Palo Alto Historical Association - A nice example of local history on the Web.

71. From Revolution to Reconstuction - an HTML project on US-history

\section{Historical Listservers}

72. Information about History related Listserv lists

73. Read History related lists

74. Genealogy

La Universidad de Kansas ha elaborado un repertorio de temas existentes en Internet que tienen relación con la Historia. Este repertorio, llamado comúnmente Indice de Kansas, se encuentra en http.//ukanaix.cc.ukans.edu:80/history y contiene novecientas ochenta y dos entradas, - sitios dicen los informáticos-, donde pueden hallarse desde estudios africanos (African Studies) hasta las últimas noticias sobre World Wide Web (WWW: WebNews). Por su gran interés se reproduce, a continuación los títulos de estas entradas.

\section{African Studies*}

Ancient Near East: ABZU

Archaeology: WWW Subject: Archaeology*

\#Archive: National Archives [NARA] by gopher [US]

\#ArchNet WWW* [US]

\#Associations: Scholarly Societies Project [CA]

\#Bibliography: ALCUIN [Contains Alex

Catalog of Electronic Texts on the Internet]

\#CLIO* [National Archives] [US]

\#Comprehensive List of WWW Sites* [US]

\#Coombsquest: WVvW Subject: Social

Sciences [US]
\#United Kingdom: Institute of Historical Research [IHR-INFO]* [UK]

\#History Departments* [US]

\#Human-Languages Page [US]

\#Index: Library: OPAC Index [US]

\#Latin America and Spain WWW [US]

\#Latin America: Latin American Studies* [US]

\#Library: Libraries available via WVvW*

\#Library: Library of Congress - Catalog and Records [US]

\#Library: Library of Congress *

\#Library: PORTICO: The British Library [UK]

\#Medieval Europe: Labyrinth: Medieval Studies Infoserver ${ }^{*}$ [US] 
\#Oceania: Pacitic Studies Facility [AU] \#Omnivore News Service* [US]

\#REES: Russian and East European Studies ${ }^{\star}$ US]

\#US: American Studies Web

\#US: American Universities Home Page* \#World Art Treasures ${ }^{*}$ [CH]

\#World History: World History* [US] 1492

Exhibition* 1755: The French and Indian War* [US]

Academic \& Research Network of Slovenia*

ACLU Reading Room

African Studies: African Art Graphics Files*

African Studies: Black/African/Development Related Information [US]

African Studies: Ethiopia Country Study* [US]

African Studies: Journal: AfricaUpdate [US]

African Studies: Mambila of Cameroon and Nigeria, Documentary History* [UK]

African Studies: Sabinet: South African Bibliographic and Information Network [ZA]

African Studies: South African Politics [ZA]

African Studies: Tunisian Gopher server [TN]

ALCEl: Association for Freedom in Electronic Interactive Communications ALIS* [DK)

Ancient Egypt on the Internet ${ }^{\star}$ [US]

Ancient Egypt: Africa: Ancient Egypt

Ancient Egypt: Alexandria*

Ancient Egypt: American Society of Papyrologists*

Ancient Egypt: Anciens documents sur l'Egypte*

Ancient Egupt: Annual Egyptological Bibliography home page*

Ancient Egypt* [US]

Ancient Egypt: Clickable image of the temple of Abu Sibel ${ }^{*}$

Ancient Egypt: Djoser Complex*

Ancient Egypt: Duke University Special Collectins Library*

Ancient Egypt: Edingburgh Ras Shamra Project $^{\star}$
Ancient Egypt: Egypt's Eastern Desert (US) ${ }^{*}$

Ancient Egypt: Egyptology Resources from the University of Cambridge *

Ancient Egypt: Egyptology [UK]

Ancient Egypt: Gesamtverzeichnis der griechischen Papyrusurkunden Aegyptens [DE]

Ancient Egypt: History of Ancient Egypt*

Ancient Egypt: Journal: ZPE: Index of the Journal of Papyrology ${ }^{\star}[D E]$

Ancient Egypt: Les obelisques* University*

Ancient Egypt: Netherlands Institute for the Near East*

Ancient Egypt: Newton Institutute Egyptology Homepage*

Ancient Egypt: Nubia Exhibits at the Oriental Institute Museum, U. Chicago*

Ancient Egypt: Papyrological resources by Gopher ${ }^{*}$ [US]

Ancient Egypt: Papyrology Collection U. of Michigan* [US]

Ancient Egypt: Papyrology [US]

Ancient Egypt: Restitutions virtuelles*

Ancient Egypt: Restoring Ancient Egyptian artifacts...by computer ${ }^{*}$

Ancient Egypt: World Art Treasures: Portraits Romains d'Egypte*

Ancient Greece: City of Athens: the archaeological and architectural ${ }^{*}$

Ancient Greece: Cornell Halai East Lokris Project (CHELP)*

Ancient Greece: Greek Architecture [US]

Ancient Greece: Greek Mythology [DE]

Ancient Greece: Hellenic Civilization Database [GR]

Ancient Greece: Journal: Didaskalia: Ancient Theatre Today [AU]

Ancient Greece: Ohio State University Excavations at Isthmia*

Ancient Greece: Pylos Regional Archaeological Project*

Ancient Greece: Rome: Greek \& Roman Cities of Western Turkey*

Ancient Greece: Thesaurus Linguae Graecae [US]

Ancient Greece: WebAcropolis [GR]

Ancient Near East: Ancient Shikhin and Sepphoris, Israel* 
Ancient Near East: Ancient Synagogues in the Holy Land - What Synagogues?*

Ancient Near East: and Palestine* [US]

Ancient Near East: Baalbek, Jbeil, and Sour Four Photos*

Ancient Near East: Baalbek/Heliopolis four photos*

Ancient Near East: Dead Sea Scrolls Exhibition* [US]

Ancient Near East: Excavations at Ciftlik (Sinop), Turkey, University of Warwick* Ancient Near East: Northeastern Turkey Archaeological Project*

Ancient Near East: Objects from Karanis in the Kelsey Museum of Archaeo$\log y(U S)^{*}$

Ancient Near East: Oriental Institute, UChicago [FTP] [US]

Ancient Near East: Radar image of Ubar and region*

Ancient Near East:Dead Sea Scrolls Exhibit*

Ancient Near East*

Ancient: Classical, Medieval, and Renaissance Achitecture and Sculpture $(\mathrm{AU})^{*}$

Ancient: Classics and Mediterranean Archaeology [US]

Ancient: Journal: Arachne. Ancient Literature and History* $\left.{ }^{*} \mathrm{IT}\right]$

Ancient: Journal: Bryn Mawr Classical Review [US]

Ancient: Journal: Electronic Antiquity: Communicating the Cla [AU]ssics

Ancient: Journal: Scholia [ZA]

Ancient: Journal: TOCS-IN: Tables of Contents Ancient History Journals [US]

Ancient: L'archeologie sous les mers [FR] Ancient: La Maison de l'Orient Mediterranean" [FR]

Ancient: More Classical, Medieval, and Renaissance Achitecture and Sculpture*

Ancient: Origins of Celtic Art [US]

Ancient: Perseus Page [US]

Archaeological reports on-line* [US]

Architectural Images* [US]

Architecture Overview of the Mediterranean Basin* [US]
Archive: Ireland: Danann Irish National Archive Server [IE]

Archive: RADIO ARCHIVE: Search Catalogue of Recorded Radio Programs [US]

Archive: UVanderbilt Television News Archive [US]

Archives and Archivists* [US]

Archives of the Peabody Institute* [US]

Archives: British ColumbiaU Archives and Records Service [CA]

Archives: Notre Dame Archives* [US]

Archives: Ohio University Archives* [US]

Archives: Project Archives on Indigenous Peoples [US]

ArchNet Gopher [US]

ArchWeb: WWW Server Nederlandse Archeologie [NL]

Art Serve* $[\mathrm{AU}]$

Art-History-related images*

Asian Studies: Chinese Communist Leaders, Voices of [AU]

Asian Studies: Buddhist Studies* [AU]

Asian Studies: CERNET: China Education and Research Network at Bejing

Asian Studies: China Research Information \& Resources [AU]

Asian Studies: China: Culture [US]

Asian Studies: China: History [US]

Asian Studies: Chinese Law database [HK]

Asian Studies: Chinese Studies [US]

Asian Studies: Journal: Echosea: Economic History of South East Asia Newsletter [AU]

Asian Studies: Journal: Mimos: Malaysia [MY]

Asian Studies: Korea [KR]

Asian Studies: Macau

Asian Studies: Malaysia

Asian Studies: Nippon Telegraph \& Telephone Infoserver ${ }^{*}$ [JP]

Asian Studies: Radar Image of the Silk Road [US]

Asian Studies: Singapore On-Line Guide [SG]

Asian Studies: South Asian/Southeast Asian Studies [US]

Asian Studies: Tibetan Studies [AU]

Asian Studies: Viet Gate 
Asian Studies: Viet-Web

Asian Studies: Vietnam Library

Asian Studies: Vietnam: Metro Multimedia Demo

Asian Studies: Vietnamese Art and monuments [US]

Asian Studies: WWW Subject: Asian Studies* [AU]

Assembly of European Gophers [SE]

Association for History and Computing * [NL]

Association: MS: American Antiquarian Society

Association: ABM: The Antiquarian Booksellers'

Association of America

Association: American Academy of Arts and Sciences

Association: American Antiquarian Society Association: American Studies Association*

Association: Association of Asian Studies* [US]

Association: International Internet Association [US]

Association: International Multimedia

Teleconferencing Consortium

Association: Kansas Humanities Council [US]

Association: Medieval Europe: Texas Medieval

Association ${ }^{\star}$ [US]

Association: National Council for the Social Studies

Association: Organization of American Historians [US]

Association: US Institute of Peace [US]

Associations: British Council* [UK]

Associations: Council for the International Exchange of Scholars

Astute: Center for Instructional Technology

Australia: Clionet: Australian History [AU] AVHRR Land Pathfinder Data Set ${ }^{*}$ [US]

BBC Networking Club* [UK]

Belgium: Belgian Academic Network* [BE]

Belgium: BELNET Research Network [BE]

Bible Gateway, Calvin College* [US]

Bible Search Facilities [US]

Bibliography: ALEX: A Catalogue of Electronic Texts on the Internet
Bibliography: Association of American University Presses [US]

Bibliography: Blackwell's Book Sellers* [UK]

Bibliography: Blackwell's Extra International Mail Order Book Service ${ }^{*}$ [UK]

Bibliography: Carnegie-Mellon University Library Catalogue Search [US]

Bibliography: Catalogues Bibliotheques [OPAC] et Autres Serveurs [FR]

Bibliography: Current Cites* [US]

Bibliography: Electronic Sources for Western European History [US]

Bibliography: General Subject Headings

Bibliography: German History Resource Guide [US]

Bibliography: Hierarchical Subject Index* [US]

Bibliography: Internet Book Shop* [UK]

Bibliography: Internet Clearinghouse: Specialized Internet Guides* [US]

Bibliography: JH-PRESS Johns Hopkins Press Pre-publication Materials [US]

Bibliography: Journals: Search Tables of Contents [US]

Bibliography: M\&M The largest bookstore in Iceland*

Bibliography: On-Line Books Index by Author* [US]

Bibliography: On-Line Books Index by Subject* [US]

Bibliography: On-Line Books: Oxford Text Archives [UK]

Bibliography: Pirrazadish: Achmaemenid Bibliography [US]

Bibliography: Portugal: Bibliografia Anual de Historia de Portugal* [PT]

Bibliography: Publishers' Catalogues* [CA]

Bibliography: Search Unesco Bibliography [EEC]

Bibliography: UnCover Periodicals Database [US]

Bibliography: Unesco Bibliographic Database [EEC]

Bibliography: Union List of Selected West European Newspapers In New York City Libraries [US]

Bibliography: US Military Institute. Reference Bibliographies [US] 
Bibliography: UWashington Publications Services [US]

Bibliography: Western European Studies [US]

Bosnia [US]

Canada: Heritage Information Network [CA]

Canada: Journal: Heritage, A Canadian History Magazine* [CA]

Canada: Louisbourg, Fortress of* $[\mathrm{CA}]$

Canada: Museum of Canadian Life*

Canada: the Canadian Resources Page* [CA]

Case Western ReserveU: Project Aurora* [US]

CCAT: Electronic Texts [US]

Center for Excellence in Education* [US]

Center for Global Communications * [JP]

Central European Environmental Libraries [Listings] [US]

Centre for Computing in the Humanities Course Materials [CA]

Centre for Humanities Computing: Oxford $W_{W W^{*}}$ [UK]

Centre for Textual Studies: Oxford WWW* [UK]

Centre National de la Prehistoire [FR]

Centre Pompidou* [FR]

CERFnet [Internet provider]

CERRO: Central European Regional Research Organization [AT]

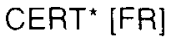

Chicago's Home Page*

Christian Coptic Orthodox Church of Egypt $^{*}$

Climateology: El Nino Data*

Climatology: CIESIN: Global Change Information Gateway [US]

Climatology: NOM: Environmental Information Services [US]

Climatology: Palynology and palaeoclimatology ${ }^{\star}$

Climatology: Radiocarbon Gopher [US]

Climatology: UNEP, Global Resource Information Database [GRID] [NO]

Cliometric Society: Economic History [US]

CPET: Projects in Electronic Texts [US]

Criminal Justice Country Profiles [US]

Croatia: CARNet: Academic and Research Network [HR]
Cyberagentes*

CyberDeals*

Cyprus [US]

Demography: Center for the Study of Population: Florida StateU [US]

Demography: Demography \& Population Studies* $[\mathrm{AU}]$

Demography: European Fertility Project 19th and 20th Century Demographic Data* [US\}

Demography: Population Index On-Line [US]

Demography: Population Studies Center at U Michigan* [USA]

Demography: Population Studies Center: UPennsylvania [US]

Demography Search the "ANUDemography Publications database» [AU]

Denmark: DENet Academic Network* [DK]

Department of Computing ${ }^{\star}$ [UK]

Department of History, University of Jyvaskyla [FI]

Direct Network Access [Internet access provider] ${ }^{*}$

Document: NAFTA: Draft of the North American Free Trade Agreement [US]

Documents: NATO: North Atlantic Treaty Organization Documents [NATO]

Documents: Relating to the Canadian Constitution [US]

Documents: Relating to the U.S. Constitution [US]

Documents: Texts of Various Constitutions of the World [US]

DOE Office of Human Radiation Experiments Home Page*

Dutch State Service for Archaeological Investigations [NL]

Early Modern Europe: 1492 Exhibit * $^{*}$

Early Modern Europe: 17th-Century Resources

Early Modern Europe: Art of Renaissance Science $^{\star}$ [US]

Early Modern Europe: Cahiers Elisabethains*

Early Modern Europe: Columbus and the Age of Discovery: An Electronic database $^{*}$ 
Early Modern Europe: Cransley Mill: Water Mills in Northamptonshire (UK)*

Early Modern Europe: Eighteenth-Century Resources*

Early Modern Europe: Eighteenth-century resources*

Early Modern Europe: Electric Renaissance* [US]

Early Modern Europe: Hartlib Papers Project ${ }^{\star}$ [UK]

Early Modern Europe: Images from the Koninklijke Bibliotheek (NL) ${ }^{*}$

Early Modern Europe: Journal: South Central Society for 18th Century Studies: Newsletter [US]

Early Modern Europe: Le siecle des lumieres dans la peinture des musees de France ${ }^{[F R}$ ]

Early Modern Europe: Leonardo da Vinci Museum

Early Modern Europe: Project Aldus Picture Gallery ${ }^{*}$

Early Modern Europe: Project Aldus: An Early Modern Virtual Library and Resource*

Early Modern Europe: Renaissance and Baroque Architecture* [US]

Early Modern Europe: Renaissance and Baroque Architecture [US]

Early Modern Europe: Renaissance Dance Archives [US]

Early Modern Europe: Renaissance Dante in Print ${ }^{\star}$ [US]

Early Modern Europe: Shakespeare Glossary

Early Modern Europe: Vatican Exhibit*

Early Modern Europe: Vermeer, Jan* [US]

EARN: European Network Welcome Page ${ }^{*}[\mathrm{CH}]$

East Asia: Indonesian Home Page [ID]

East Asia: Japan Center at Stanford

East Asia: Japan Information: History

East Asia: Japan: Art ${ }^{\star}$

East Asia: Journal: Wired: Net News From Singapore* [SG]

East Asian Hongkong Internet Gateway \& Services [HK]

East Asian: History of Japan [JP]

East Asian: Hong Kong law database* [HK]
East Asian: Images from Japan

Ecolab Gopher

Educational Online Services [US]

Educational Resources Information Center [also known as ERIC] [US]

Educational Sources [K12] On-Line* [US]

EDUCOM Server ${ }^{*}$ [US]

EE-Link, the Environmental Education Gopher [US]

ELAG Seminar Papers [NL]

Employment Opportunities and Job Resources on the Internet* [US]

English and Literature Gopher: UPennsylvania

English Literary Texts* [US]

Erasmus: Arts Faculty, ULeuven* $[B E]$

Estonia: EENet Academic Network ${ }^{*}$ [EE]

Estonia: Estonian Schoolserver ${ }^{*}$ [EE]

EUROPA*: Gateway to the European Union [LU]

European Home Page * [EU]

Finland and Estonia Online ${ }^{\star}[\mathrm{FI}]$

Finland: Tampere: Materials for the History of* $[\mathrm{FI}]$

France: Armada de la Libert\&eacute; [FR]

France: French Literary Texts [SE]

France: Library: IRCAM: French Libraries OPAC* $[F R]$

France: Ministry of Culture, France: Links to Various Sites [FR]

France: Rennes U: French Discussion List Archives [FR]

FUNET: Finland [FI]

Medieval Europe: Medieval Studies* [US]

Genealogy: Everton Genealogy Page* [US]

Genealogy: Genealogy Gopher [US]

Genealogy: Genealogy Help and Guides* [US]

Genealogy: Genealogy Toolbox* [US]

Germany: Eastern German Studies Group* [US]

Germany: EUnet in Germany [DE]

Germany: Euronet* [DE]

Germany: Index of German Nobility* [DE] Germany: Modern: Passau Under the Third Reich, City Tour ${ }^{*}$ [DE]

Germany: Modern: White Rose* [US]

GESIS: German Social Science Infrastructure Services 
GHETA: Historical Archives at Gronigen [by gopher] [NL]

GHETA $^{*}[\mathrm{nl}]$

Gilbert and Sullivan Archive * [US]

GIS and Remote Sensing for Archaeology: Burgundy, France [US]

GlasNet [SU]

Global Jewish Information Network System* [IL]

Global Network Academy, Inc. *[US]

Global Network Navigator* [Also known as GNN] [CA]

Gopher Jewels [US]

Gopheur: Instruments de Recherche en Ligne [CA]

GRADUATE STUDENTS*: History Graduate Students Association at Uindiana at Bloomington [US]

Grants: IRIS: Funding Source Data Base [US]

Grants: MOLIS: Minority Online Scholarship Service [US]

Grants: NIH Guide to Grants and Programs [US]

Grants: NSF Abstracts of Awards [US]

Grants: NSF Bulletin [US]

Grants: NSF Publications Overview [US]

Grants: NSF Publications [US]

Grants: NSF STIS Topics System [US]

Grants: NSF: STIS Gopher System [US]

Graphics: Digital Image Center Image Collections

Graphics: Eastman Kodak* [US]

Graphics: Fine Art Archive* [JP]

Graphics: GALLERY V: Berea College Museum* [US]

Graphics: Icon Browser: PisaU* [IT]

Graphics: Icons [US]

Graphics: Images, Icons, Flags* [US]

Graphics: UUtrecht, Image Retrieval and Information System [NL]

Graphics: WWW Cyberview 3d Document Generator*

Greece: Academic and Research Center [GR]

H-Albion [US]

$\mathrm{H}$-Net Archives [US]

Heidelberg History Archives* [DE]

Historical Documents [US]

History [University of Toronto]
History Archives - gopher [US]

History at the English Server* [US]

History Graduate Students Association at Indiana

University at Bloomington* [US]

Holocaust and Fascism Archives* [CA]

Holocaust Index

Holocaust Memorial Musem. Washington D.C.

Holocaust: A Cybrary of the Holocaust* [US]

Humanistisk Datasenter i Bergen [NO]

Humanities Computing Center UCSB [US]

Humanities Computing Resources UToronto [CA]

Hungary: Hungarian Gopher [HU]

Hypergarden* [US]

Military: World War II FAQ [US]

IATH: Institute for Advanced Technology in the Humanities* [US]

Images of My War* [US]

Index of German Nobility ${ }^{\star}$ [DE]

Index: GARR-NIS*: General WWW Access to Italian Network Information Service [IT]

Index: Ancient Greece: Hellenic Resources

Index: Arts \& Sciences Gopher: Washington University [US]

Index: Arts and Humanities* [US]

Index: Cisco Educational Archive* [US]

Index: DA-CLOD: Web LinksDatabase* [US]

Index: EINet Arts and Humanities Register ${ }^{*}$ [US]

Index: EINet Directory Service* [US]

Index: Entering the World-Wide Web: A Guide to Cyberspace* [US]

Index: European Historical Sites by Gopher [NE]

Index: France: GRENET ${ }^{*}$ : The Doyen of French Internet Sites [FR]

Index: Geographic Information Server* [US]

Index: Guides to the Internet [US]

Index: HELP: Internet Assistance [US]

Index: History Information Server [US]

Index: Interactive RFC Index* [US]

Index: International History and Politics Infoserver ${ }^{\star}[\mathrm{CH}]$ 
Index: Internet Guides and Information Services [US]

Index: Internet Index on SilverPlatter's Home Page * [US]

Index: InterNIC Directory of Directories

New Listings

Index: InterNIC Directory of Directories

Index: Interpath ${ }^{\star}$ [US]

Index: Journal: Internet Scout Report* [US]

Index: List of Discussion Lists of Interest to Historians.

Index: Microsoft's Internet Assistant" Index: NISS Information Gateway [UK]

Index: Nova-Links Internet Search Server* [US]

Index: PEG Internet Assistance [US]

Index: PEG Libraries [US]

Index Subject-Oriented Internet Resource

Guide [CA]

Index: Wiretap Server [US]

Index: Yahoo: Alternative World Wide

Web Subject Index*

Index: Yanoff Guide [US]

India, Education and Research Network [IN]

India Network Gopher

Indigenous Peoples: Ancient Cultures of

the Western Hemisphere [US]

Indigenous Peoples: Canadian Indian,

Inuit and Mitis cultures (CA)*

Indigenous Peoples: Canandaigua Treaty of $1794^{*}$

Indigenous Peoples: Center For World Indigenous Studies [US]

Indigenous Peoples: Maxwell Museum of Anthropology*

Indigenous Peoples: Native American

Issues and Culture [US]

Indigenous Peoples: NativeNet Information Network* [US]

Indigenous Peoples: Oneida Indian Nation Indigenous Peoples: Russian Church and

Native Alaskan Cultures [US]

Indigenous Peoples [US]

Indigenus Peoples: Ethnic Studies at USC*

Information Network of Kansas Guest

Access [also known as INK] [US]

INS Information Services* [US]
Institut National de Recherche en Informatique et Automatique [also known as INRIA] [FR]

Institute for Advanced Technology in the Humanities [IS]

Institute for Social Studies, UWarsaw* [PL]

Instituto di Elaborazione dell'informazione, Image Processing Service* [IT]

International Centre for Distance Learning [UK]

International Research and Exchanges Board [US]

International Studies Graduate Institute, UGeneva* $[\mathrm{CH}]$

Internet Discussion Lists of Interest to Historians

Internet Presence and Publishing Corporation * [US]

Internet Tools [US]

Internet Training Sources *

Ireland On-Line ${ }^{*}[\mathrm{IE}]$

Ireland: IEunet [IE]

ISMC Graphics Interface to ISMC* [US]

Islam: Association: Middle East Studies Association [US]

Islam: Islamic Architecture in Isfahan* [UK]

Islam: Islamic Resources Gopher

Islam: Middle East Center: Univ. of Utah [US]

Islam: Middle East-North Africa Internet Resource

Guide [Roberts] [US]

Islam: Middle Eastern Gophers [US]

ISnet Information Services [IS]

Israel: Jerusalem Mosaic* [IL] [IL]

Israel: Jerusalem One: Israeli Information Server [IL]

Israel: Jewish Information Network System * [IL]

Israel: Official and Government Sources [IL]

Israel's Daily Press and Broadcast services [IL]

Italy: Connections to All Gophers in Italy [IT]

Italy: Italian Language Mailing List for Web Users* [IT]

Italy: Italian Literary Texts* [IT] 
Italy: Library: Italian Libraries OPAC [IT] Italy: Library: OPAC: Italy [by gopher] [IT] Italy: Library: OPAC: Italy * [IT]

Italy: Window on Italy ${ }^{*}$ [US]

Italy [IT]

Jewish Resources Register ${ }^{\star}$ [US]

Journal for Humanists [NO]

Journal: Essays in History [US]

Journal: Academe This Week

Journal: Edupage* [UK]

Journal: International Philosophical Preprint

Exchange [also known as IPPE] [US]

Journal: Internaut: Magazine for Internet users $^{*}$ [US]

Journal: IPCT: An Electronic Journal for the 21st Century [US]

Journal: Judaica: Jewish Studies [US]

Journal: Mother Jones* [US]

Journal: REACH: Computer in the Humanities Electronic Journal [US]

Journal: Scholar [US]

Journal: Software and Courseware OnLine Reviews [select vt1 00/c/2] [AU] Journal: TOC of history journals

Journal: Trinity Coliege Electronic Journal* [US]

Journal: Wilson Library Bulletin [US]

Journals Archive at CICNet [US]

Journals in History and Geography [US]

Journals in History [US]

Journals in Interpersonal Computing \&

Technology [US]

Journals in Military Science [US]

Journals in the Social Sciences [AU]

Journals: Association of Research Libraries:

Directory of Electronic Journals ${ }^{\star}$ [US]

Journals: $\mathrm{CIC}$ Electronic Journals

Collection [CIC-EJC] [US]

Medieval Europe: Latin Texts: Recentiores [US]

Karolinska Institute * [SE]

Kassandra Project*

Medieval Europe: Robert Duncan's Medievalist's E-Address Book

Language: ARTFL* by hhtp [US]

Language: ARTFL by gopher [US]

Language: CELIA: Computer Enhanced

Language Instruction Archive [US]

Language: Chinese WWW* [SG]
Language: France \& French Language* [US]

Language: French Lessons on the Web* [US]

Language: Italian Language Lessons* [US]

Language: Journals in Languages [US]

Language: Language Bank of Swedish* [SE]

Language: Language Bank: Sproekbanken at GotenborgU [SE]

Language: Languages: Less CommonlyTaught Languages Index [US]

Language: LeedsBit: TrueType fonts for Czech, Polish, Hungarian, Greenlandic, Turkic, etc.

Language: Library: Project Runeberg: Scandinavian Texts [SE]

Language: Linguistica Informatica [ES]

Language: Melange: On-Line Language Tutorials* [SG]

Language: Persian text viewer* [US]

Language: Welsh Language and Culture Archive [US]

Latin America: Argentina: Government Gopher [AR]

Latin America: Bolivia* Experimental Home Page [US]

Latin America: Connection Collection* [US]

Latin America: ECUANET: Ecuador Net [EC]

Latin America: History \& Struggle of Cuba* [US]

Latin America: INFO-SOUTH: The Latin American Information System [US]

Latin America: Mexico: Culture and Society* [US]

Latin America: Peru: Red Cientifica Peruana* $[P E]$

Latin America: Red Academica Uruguaya [RAU] [UY]

Latin America: Red Nacional de Pesquisa Central* $[B R]$

Latin America: Reuna Red Universitaria Nacional [CL]

Latin America: UTexas-LANIC: Latin American

Information Center [US]

Learned Information Net* [UK]

Library: Atrium: Bibliotheques de I'Universite de Montreal [CA] 
Library: Bibliotheques de I'USHS [Strasbourg] [FR]

Library: Carrie: An Electronic Full-Text Library [US]

Library: Center for Research Libraries* [US]

Library: Electronic Text Center at UVirginia WWW* [US]

Library: Electronic TextCenter at UVirginia Gopher [US]

Library: General Vatican Library*

Library: Healey Library Gopher [US]

Library: Information Servers* [US]

Library: Internet Public Library: School of Information and Library Studies at the University of Michigan [US]

Library: Library of Congress Exhibitions On-Line [US]

Library: Lyndon B. Johnson Presidential Library [US]

Library: MARVEL - Foreign Government Resources ${ }^{\star}$ [US]

Library: MARVEL: Library of Congress Gopher [US]

Library: On-line Books*

Library: Radcliffe and Bodleian Libraries* [UK]

Library: Southeastern Library Network [US]

Library: Special Collections: TulaneU* [US]

Library: Special Collections: $U$ of Houston* [US]

Library: Special Collections: UVirginia [US]

Library: Texas State Electronic Library [USA]

Library: URutgers: Center for Electronic Texts in the Humanities* [US]

Library: UWaterloo Electronic Library [CA]

Library: Vatican Library*

Library: Virtual Library Information: Catriona [UK]

Library: Waterloo Online Books Initiative Archive [CA]

Library: Waterloo Univ. Electronic Library* [CA]

United Kingdom: Modern Records Centre: University of Warwick Library [UK]
Military: WWII Underground Factory in Czech Republic* [US]

Listserv User Guide [version 2] [NL]

Literature at the English Server: Poetry* [US]

Literature at the English Server: Drama* [US]

Literature at the English Server: Fiction* [US]

LoQtus: The Quotations Web Page* [US]

Louvre [une petite visite guidee de Paris] ${ }^{\star}$ [FR]

Luxembourg: Education and Research Network* [LU]

Mailbase ${ }^{*}$ [UK]

Malin: Historical Archives [ftp ukanaix.cc. ukans.edu co pub/history] [US]

Maps: Earth Images from NASA* [US]

Maps: Earth Images from NASA Gopher [USA] [for ftp: images.jsc. nasa.gov login=anonymous]

Maps: Historical Map Collection - U. of Georgia* [US]

Maps: NASA, Johnson Space Center, Earth images [US]

Maps: Perry-Castaneda Collection* [US]

Maps: Rank Xerox Public Web Maps

Maps: The U.S. Geological Survey [US]

Maps: Xerox PARC Map Viewer ${ }^{\star}$ [US]

Maritime History ${ }^{*}$ [SE]

Maritime Museums ${ }^{\star}$ [SE]

Maritime Museums [UK]

Massachusetts Education Computer Network [US]

Material Culture Resources

Medieval Europe: All Hallows parish church, Nottingham*

Medieval Europe: American University FTP Archive

Medieval Europe: Arthuriana*

Medieval Europe: Associations: American Branch of the Richard II"*

Medieval Europe: Atrium: Medieval Documents* [US]

Medieval Europe: Augustine, Saint: Materials from an on-line seminar* [US]

Medieval Europe: Avalon* [Arthurian Lore]

Medieval Europe: Beowulf Project [UK] 
Medieval Europe: Bryggen Runic Font [110k] [for Macintosh] [NO]

Medieval Europe: CANTUS - Database for Gregorian Chants for the Divine Office [US]

Medieval Europe: Curia: Irish Text Project $^{\star}[\mathrm{IE}]$

Medieval Europe: Dante Project* [US]

Medieval Europe: Dante: Divina Commedia* Medieval Europe: Dante: Vita Nuova*

Medieval Europe: DILS Project

Medieval Europe: DScriptorium: medieval manuscripts [US]

Medieval Europe: Exeter Book*

Medieval Europe: Gregorian Chant* [US]

Medieval Europe: Guide to Early Church Documents* [US]

Medieval Europe: History [FI]

Medieval Europe: Horae Beatae Virginis Mariae, secundum usum Romanus*

Medieval Europe: Hwft! Old English in Context*

Medieval Europe: Index of WWW Medieval Resources* [US]

Medieval Europe: International Reynard Society*

Medieval Europe: Jour dou jugement*

Medieval Europe: Journal: American Academy of Research Historians of Medieval Spain Newsletter

Medieval Europe: Journal: Bryn Mawr Medieval Review [US]

Medieval Europe: Journal: Exemplaria: Theory in Medieval and Renaissance Studies* [US]

Medieval Europe: Latin Text Archives at Wiretap [US]

Medieval Europe: Latin Text Archives at Libellus [US]

Medieval Europe: Latin: Council of Remiremont ${ }^{*}<$ US $>$

Medieval Europe: Les tres riches heures du Duc de Berry*

Medieval Europe: Manuscript Study: Basic Information*

Medieval Europe: Medieval Iberian Archives [US]

Medieval Europe: Medieval Studies at Univ of Connecticut*
Medieval Europe: Middle English Dictionary ${ }^{\star}$

Medieval Europe: Mittelalterliche Kirche [UGiessen, DE]

Medieval Europe: Mittelalterliche Universitaeten [UGiessen, DE]

Medieval Europe: Oxford Gateway to Medieval Studies*

Medieval Europe: Piers Plowman Project ${ }^{*}$

Medieval Europe: Rune Typology Project [NO]

Medieval Europe: Saint Patrick: Autobiography [US]

Medieval Europe: Search Runic inscriptions [NO]

Medieval Europe: Sigtuna Museum ${ }^{\star}$ [SE]

Medieval Europe: Troms\&oslash; Museum [NO]

Medieval Europe: U. of York, Centre of Medieval Studies*

Medieval Europe: Vatican Library MSS Exhibit*

Medieval Europe: Viking Materials ${ }^{\star}$ [SE]

Medieval Europe: Viking Navy* [SE]

Medieval Europe: Viking Network* [NO]

Merit Network Information Center [US]

Military History Repository* [US]

Military History: Gulf War - Diary of Judy Koren* [US]

Military History: Gulf War - Diary of Robert Werman* [US]

Military History: Gulf War Photo Gallery* [US]

Military History: MIL-HIST INformation Server* [US]

Military History: Operation Desert Storm Debriefing Book* [US]

Military: ADFA: Australian Defense Force Academy

Military: Australian Defence Force Academy,

Minnie* [AU]

Military: Canadian War Museum*

Military: Cuban Missile Crisis* [US]

Military: Defense Simulation Internet [DSI] Server ${ }^{\star}$ [US]

Military: Desert Storm Picture Gallery* [US] Military: E-HAWK Electronic Headquarters for the Acquisition of War Knowledge* [US] 
Military: Graz Arsenal* [NL]

Military: Jane's Online*

Military: Korean War Project* [US] [US]

Military: POW/MIA Database. Library of Congress* [US]

Military: Pre-Modern Military History ${ }^{\star}[$ US]

Military: Salzburg: 1945-1955: Liberators and Occupiers ${ }^{\star}$ [AT]

Military: Subject Tree [UK]

Military: Sunzi: The Art of War [Chinese] [SG]

Military: The Fall of Singapore [SG]

Military: The Warsaw Uprising*

Military: US Army Military History Institute* [US]

Military: Warsaw Uprising, $1944^{*}$ [US]

Military: World's Elite Combat Units* [SE]

Military: WWII, D-Day* [US]

Military: WWII net, 1933-45 [US]

Modern Europe: Tito, Jozip Broz [18921980] [SI]

Modern Europe: Urban History: Wolverhampton [UK], 1827-1901 [UK]

Modern Europe: Virtual Museum in Milano: Monumentale Cemetery of Milano [IT]

Modern Europe: Virtual Venice by MultiMedia WWW PC [IT]

Monet Exhibit* [US]

Musee des Arts et Metiers of Paris* [FR]

Museum Service* [Reading UK]

National Distance Learning Center [US]

NCSA What's New Page* [US]

NearNet Arts and Humanities Register [US]

Netherlands [NL]

Netherlands Historical Data Archive by gopher [NL]

Netiquette* [US]

Network for Education and Research* [LU]

New Zealand [NZ]

New Zealand: A Brief History [NZ]

NLneVNLUUG* Netherlands Network [NL]

Norway: Computing Center for the Humanities [NO]

Norway: Gopher Server [NO]

OASIS: Leiden University Publications [NL]

Oceania: Journal: Pacific Islands Group Newsletter [AU]
Oceania: Journal: PAMBU: Pacific Manuscripts Bureau Nwsltr [AU]

Oceania: Philippine Literature ${ }^{\star}$ [US]

Old Postcard Views* [SE]

On-Line Book Initiative [US]

On-Line Literary Research Tools*

Palladio Image Archive [US]

Paris Libere!* [US]

Paris WWW* [US]

Peabody Museum: Search of All USC\&G Place-Names [US]

PEG Welcome Page [US]

Planet Earth* [US]

Poland Home Page

Poland: The Tatra Mountains Virtual Tourist*

Political Science* [US]

Portugal: Portuguese root gopher [PT]

Prairienet [US]

Prehistory: Aegean Prehistory Course

Prehistory: ALERT!! —Paleolithic art from Foz Coa, Portugal, in danger*

Prehistory: Archaeological Research at Oslonki,

Poland* [US]

Prehistory: Art Pariital ${ }^{*}$

Prehistory: Cave Paintings: New Discovery* [FR]

Prehistory: Departmento de Prehistoria y Arqueologia

Prehistory: Journal: Old World Archaeology Newsletter [US]

Prehistory: Megaliths of Morbihan [UK]

Prehistory: New World Archaeology* [US]

Prehistory: Vallon-Pont-d'Arc [Ard\&egravejche] [FR]

Principia Cybernetica Project ${ }^{\star}[\mathrm{BE}]$

RA: Historical Archives* [ftp.msstate.edu cd/pub/docs] [US]

REES: EX-USSR: Materials on PostSoviet States* [US]

REES: Friends and Partners: RussianAmerican Information Server* [US]

REES: Journal: Slavic Review* [US]

REES: Khazaria Information Center ${ }^{*}$ [US]

REES: Lithuanian Nationa! Page* WWW [LT]

REES: Post-Soviet \& Eastern Europe [US] REES: Russian \& European Network Information Center [US] 
REES: Sovam Teleport- Ukraine

REES: Soviet Archive Exhibit*

REES: Soviet Archives Exhibit [US]

REES: St Petersburg Pictures Gallery ${ }^{*}$ [SU]

REES: Treasures of the Czars, 1613 to 1917 [US]

REES: Window to Russia* [SU]

Reference: Bartlett's Familiar Quotations* [US]

Reference: Guide to Writing HTML Documents* [US\}

Reference: Michael Moncur's Quotations Page*

Reference: Reference Desk [US]

Reference: Reference Desks, Global [US]

Reference: Rodman Thesaurus [US]

Reference: Roget'sThesaurus [1911 ed.] [US]

Reference: University of Illinois Grammar Guide [US]

Reference: Wilson, H.W. Company [US]

Reference: WWW: Writing HTML documents* [CA]

Reuben and Edith Hecht Museum, Haifa University*

Riddle Project* [NL]

Romania: Home Page* [RO]

Romania: Information [RO]

Rome: Aeminium \& Roman Portugal (Museu Nacional de Machado*

Rome: Age, Gender and Status Divisions at Mealtime in the Roman*

Rome: Amphitheatre d'El-Jem (Thysdrus); an overview*

Rome: Amphitheatres romains de Tunisie (Web reprints of two ${ }^{*}$

Rome: Ancient History Course*

Rome: Ancient Landscape of Burgundy: Applying GIS and Remote*

Rome: Archaeological Heritage of Southern Italy ${ }^{\star}[\mathrm{IT}]$

Rome: Archaeological Museum of Cagliari* [IT]

Rome: Archaeology in Campania (Istituto di Cibernetica, CNR, Napoli)*

Rome: Art and Archaeology of Ancient Italy and the Provinces of Rome* [US]

Rome: Art and architecture in Turkey ${ }^{\star}$
Rome: Braga (the Roman Bracara Augusta), Portugal ${ }^{*}$

Rome: Centre for East Roman Studies, University of Warwick*

Rome: Centre Pierre Paris, incl. Projects in Roman Tunisia, from*

Rome: Centro Ricerche Informatiche per i Beni Culturali (Pisa):*

Rome: City and Environs of Nimes, France; art and architecture*

Rome: City of Lincoln Archaeology Unit (Brayford Wharf East, 1982*

Rome: City of Trier, Germany; art and architecture*

Rome: Conimbriga: a Roman town in Portugal*

Rome: Cyprus Tourist Page, including Roman sites and museum material*

Rome: Department of Archaeology, York University*

Rome: Early Church Documents - ca 96150 A.D. * [US]

Rome: Egeria's 4th c. Description of the Liturgical Year*

Rome: Excavation at the Romano-British Settlement at Broughton*

Rome: Excavation at the Romano-British Settlement at Pasture Lodge*

Rome: Excavations at a Roman fort on the River Tyne, South Shields, *

Rome: Glasgow University Archaeology Research Division*

Rome: Golfo Porte Aperte: Sites Around the Bay oif*

Rome: Gozo (Malta) Project \& Insite Project: U. Bristol, Cambridge,"

Rome: Grand amphitheatre d'El-Jem (Web reprint from J.-C. *

Rome: Hadrian's Wall Leisure \& Tourism Guide* $^{*}$

Rome: Imperium Romanorum

Rome: Laxton Village Survey and Excavation*

Rome: Leptiminus (Tunisia) Archaeological Project ${ }^{\star}$

Rome: Library: Project Libellus [US]

Rome: List of Roman and Byzantine Emperors with accompanying map*

Rome: Newstead Project: A Roman fort in Scotland [UK] 
Rome: Origins of Celtic Art*

Rome: Philodemus Project: Scrolls from the Villa dei Papiri, *

Rome: Pompeii Forum Project, University of Virginia*

Rome: Pompeii: Roman Society and Culture in Microcosm; a course at ${ }^{*}$

Rome: Roman cadastration in Britain, and ancient land management, $U$, *

Rome: Roman City of Volubilis, Morocco*

Rome: Roman fort at Newstead, University of Bradford*

Rome: Roman Law* [DE]

Rome: Roman Mosaic found at Vichten (Musee National d'Histoire et ${ }^{*}$

Rome: Roman Palace in ex-Yugoslavia [US]

Rome: Roman Republic: Warfare and Tactics* [US]

Rome: Roman resources*

Rome: Roman Scotland: an exhibition of the Hunterian Museum, Glasgow*

Rome: Roman town of Aquincum, Hungary*

Rome: Rome's Ruins, Learning to Read [US]

Rome: Salerno Porte Aperte: Brief illustrated descriptions of sites in ${ }^{*}$

Rome: Shipwrecks (including Classical wrecks) from the Mediterranean*

Rome: Terracotas Arquitectonitas del Museo Arqueologico Nacional de*

Rome: Theatre of Dionysos \& Odeion of Herodes Atticus, *

Rome: Trent \& Peak Archaeological Trust, The University of Nottingham*

Rome: Trent Valley Survey*

Rome: U. Bristol, Visualisation of Landscapes Project, Gubbio Basin, *

Rome: Une banque d'images sur El-Jem*

Rome: University of Lecce:*

Rome: Winterstoke Research Project, University of Bristol*

Rome: World Art Treasures: Portraits Romains d'Egypte*

Rome: Wroxeter Hinterland Project, University of Birmingham Field*

Royal Holloway College - Art* [UK]

Sardinia: The History of Sardinia* [IT]

Scholarly Electronic Conferences on History [Ninth ed.] [US]
Science: ASAPWeb: History of Science and Techology* $[\mathrm{AU}]$

Science: Australian Science Archives Project $^{*}[\mathrm{AU}]$

Science: Early Instruments of the Institute of Physics of Naples* [IT]

Science: Greek Mathematics \& Its Modern Heirs [US]

Science: History of Astronomy Working Group of the German Astronomical Society

Science: History of Australian Science and Technology* [AU]

Science: History of Chinese Mathematics* [US]

Science: History of Computing and Data Processing $^{*}[\mathrm{AT}]$

Science: History of Mathematics Archive* [UK]

Science: History of Medicine Archives* National Library of Medicine [US]

Science: History of Science at Florence* [IT]

Science: History of Science Register* [US]

Science: History of the Light Microscope* [US]

Science: Jesuits and the Sciences, 1600 1800* [US]

Science: Journal: HASN: History of Australian Science Newsletter [AU]

Science: Journal: HOST: History of Science and Medicine [US]

Science: Library: National Library of Medicine ${ }^{*}$ [US]

Science: National Library of Medicine History of Medicine Archives* [US]

Science: On-line Typography Museum [US]

Science: OpenNet: US Department of Energy Documents* [US]

Search Engine: CUSI: Configurable Unified Search Engine*

Search Engine: EIT: Wide-Ranging WWW SeNer* [US]

Search Engine: Four11 Online User Directory ${ }^{*}$ [US]

Search Engine: HARVEST: Information Discovery and Retrieval*

Search Engine: INTERNET SLEUTH: Internet Tools [US] 
Search Engine: Netlink: Internet Search Server [US]

Search Engine: VERONICA Search System [US]

Search Engine: Virtual Reality Xplorer $v$. $1.1^{*}[\mathrm{IT}]$

Search Engine: Visual Arts Search

Engine* Search Engine: WAISGATE Directory ${ }^{\star}$ [US]

Search Engine: WAISGATE Gopher Search Systems* [US]

Search Engine: WAISGATE WAIS

Search Systems* [US] Search Engine:

WAISGATE WWW Search Systems* [US]

Search Engine: WWW: WebCrawler Index [US]

Search Engine: X500 Group at BrunelU* [CA]

Slovakia: [SANET] [SK] Slovakia: EUnet in Slovakia [SK]

Slovakia: Slovakian Republic Archive* [SK]

Social Science Data Center [UVA] [US]

Social Science Information Gateway ${ }^{\star}$ [UK]

Spain: Embassy of Spain* [CA]

Spain: Hispanic Studies Archive [US]

Spain: Interactive Map* [ES]

Spain: Library: Universidad Complutense [Madrid]* [ES]

Spain: RedIRIS, FUNDESCO* [ES]

Spain: Spanish and Portuguese Studies [US]

Spain: Spanish Embassy in Canada [CA]

Spider Web Server* US Navy [US]

SUNET EuroGopher [SE] SURF: Silicon Graphics WWW Server* [IE]

SURFnet InfoServices* [NL]

Sweden: Swedish [and Scandinavian] History... [SE]

Swedish Social Science Data SeNice* [SE]

Switzerland: EUnet in Switzerland $[\mathrm{CH}]$

Switzerland: Interactive Map* $[\mathrm{CH}]$

Switzerland: Swissinfo $[\mathrm{CH}]$ Weather World from The Daily Planet* [US]

Theology and Computing [UK]

TitleNet: Book Publishers Catalogs [US]

Turkey: Armenian Research Center: UMichigan Dearborn*
Turkey: Tubitak Srdc: Information SeNer Ankara [TR]

Turkey: Turkey in Pictures [TR] ${ }^{*}$

U Bergen, Humanistisk datasenter ${ }^{*}$ [NO]

UCalifornia at San Diego: Social Sciences Data Collection* [US]

UGoteborg: Swedish Social Science Data Service ${ }^{*}$ [SE]

Ultralab* [UK]

UMinnesota, International Study and Travel Center [11148] [US]

United Kingdom:: Collection of links to England $^{*}$ [US]

United Kingdom: Archives: National Register of Archives Guides [AU]

United Kingdom: Interactive Map* [UK]

United Kingdom: Library: United Kingdom Library Gopher SeNers Listing [UK]

United Kingdom: Modern: Rural History Centre* $^{*}$ Reading, UK]

United Nations and Other International Organisations ${ }^{*}$ [US]

University Home Pages* [US]

UPennsylvania, Social Science Computing ${ }^{\star}$ [US]

URochester: Department oF History*

US Colonial: Grove Street Cemetery*

US Colonial: Interpreting Activity Areas*

US Colonial: Mayflower Hill Project*

US Colonial: Oneida Treaties Project ${ }^{*}$

US Colonial: Plimoth Plantation.

US: 1935-1945: Colored Photo Collection* [US]

US: African-American Mosaic

US: Atro-American: The Promised Land [US]

US: American Memory - National Digital Library* [US]

US: American Revolution [NL]

US: American South Internet Resource Center

US: Ancestry: Religion, Death and Culture in Central Appalachia* [US]

US: Archaeology of the Northeastern United States*

US: Armadillo: The Texas Studies Gopher [US]

US: Black Studies, Univ. Missouri-St. Louis Library [US] 
US: Budget, U.S. National: Full Text [US]

US: BURLINGTON*: The Historic City of Burlington, New Jersey [US]

US: California Missions Interactive*: An Internet Field Trip [US]

US: Census 1990* [US]

US: Census Data, Bureau of the Census*

US: Census Data [US]

US: Census for $1850,1880,1920$ [US]

US: Civil War: 35 Days To Gettysburg, A Diary $^{*}$ [US]

US: Civil War: Archive, 1861-1865* [US]

US: Civil War: Augustana College Library Projects* [US]

US: Civil War: C.S.A.documents [US]

US: Civil War: Civil War Medicine* [US]

US: Civil War: Collection of J.C Cohen Letters* [US]

US: Civil War: Diaries from Pennsylvania and Virginia* [US]

US: Civil War: Diary of Carrie Barry* [US]

US: Civil War: Doctor's Report* [US]

US: Civil War: Excerpt From the Address of Captain Joseph A. Moore* [US]

US: Civil War: Gettysburg Address Exhibit* [US]

US: Civil War: Letter in Reply to General Johnson* [US]

US: Civil War: Letter to President Davis from General Lee* [US]

US: Civil War: Letters from an lowa Soldier in the Civil War*

US: Civil War: Pennsylvania \& Virginia

US: Civil War: Pennsylvania and Virginia Newspapers* [US]

US: Civil War: Photographic Collection* [Large] [US]

US: Civil War: Recollections of a Virginian in the Mexican, Indian, and Civil Wars [US]

US: Civil War: Timeline: Events and Photographs* [US]

US: Civil War: Vast Sea of Misery-Field Hospitals* [US]

US: Civil War: Walt Whitman's Notebooks ${ }^{\star}$ [MSS, Full Text] [US]

US: Colonial: World of Benjamin Franklin* [US]

US: Congressional Quarterly Gopher [US]

US: Constitution, AS ENGROSSED*
US: Declassihed Documents Relating to J. F. Kennedy* $[\mathrm{CH}]$

US: Department of Education* [US]

US: Department of State Foreign Affairs Network [DOSFAN] [US]

US: Dept. of Commerce: Economics and Statistics Administration [US]

US: Ecolab Gopher Archaeology of the Southeastern United States [US]

US: Emma Goldman Papers Project [US]

US: FDIC: Bank Data 1934- [US]

US: Federal Job Opportunities [US]

US: FEDIX: Federal Funding Programs Information Exchange [US]

US: General Accounting Office Reports [US]

US: Government Locator SeNice [US]

US: Heritage: Kansas Genealogy and Family and Local HistoN, [US]

US: Historic American Building SuNey

US: Historical Photographs*

US: Indiana Historical Society [US]

US: Journal: American Historical Review* [US]

US: Kansas State Historical Society [US]

US: Life Histories - Oral History Collection from the 1930's* [US]

US: Mark Twain on the Philippines [US]

US: McKinley at the Pan American Exposition, 1901* [Films] [US]

US: Military: BYRD: Historical Archives [ftp byrd.mu.mvnet.edu] [US]

US: Motion Pictures, Early History- 1897$1916^{*}$ [US]

US: Museum of the City of New York Online*

US: National Agricultural Library*

US: National Archives' Motion Picture Archives [ftp cu.nih.gov] [US]

US: National Archives [US]

US: National Space Agency Historical Archives* [US]

US: Navy Policy Book [US]

US: New York City, 1898-1906* [Films] [US]

US: New York State Archives \& Records

Administration [US]

US: Oregon State Archives*

US: Product: State Product Tables [US] 
US: Regional Oral History Project: UC Santa Cruz [US]

US: San Francisco, 1897-1916* [Films] [US]

US: Science: Faces of Science: AfricanAmericans in the Sciences* [US]

US: Sketch of a three years travell in South America, California, and Mexico [1848-1851 ] [US]

US: Staunton, Shenandoah Valley VA [2] $]^{\star}$ [US]

US: Staunton, Shenandoah Valley VA [1]* [US]

US: Supreme Court decisions, 1989-94* [US]

US: SW Pennsylvania Heritage Preservation Commission*

US: Texas Metronet [US]

US: Texts of Historic Speeches [US]

US: Texts of Treaties and International Covenants [US]

US: Vietnam Era FTP SeNer [US]

US: West Virginia Gopher Site [US] [US]

US: Zipcode Database [US]

USan Diego: Department of History [US]

Usenet Access * [US]

UTennessee Tech: History Resource [US]

UToronto Instructional and Research Computing Group* [CA]

Wayne StateU: Department of History [US]

Weather for the State of Kansas [US]

Weather for the United States [US]
Weather: Comprehensive Weather SeNer [US]

Women's Studies Database [US]

World Art Treasures*

World Factbooks, 1990-1992 [US]

World History: Journal: World Heritage [US]

World History: New England Regional World HistoN Association Archives [US]

World History: World Cultures to $1500^{\star}$ [US]

World History: World Feudalism checklist [Haines Brown] [US]

World History: World History Documentary Archives [US]

World History: World History Gateway* [US]

World History: World Systems Archives [US]

World-Wide Art Resources - Galleries and Exhibitions* [US]

WWW CyberWeb: resources for WWW developers*

WWW Development*

WWW MultiMedia PC - v. 2.0 Upgrade Distance Education

WWW Software

WWW Sports Home Page* [UK]

WWW STATS software [C-shell] $]^{*}$

WWW STATS software [perl]

WWW Support* $[\mathrm{CH}]$ WWW: Address as of 10 February $1995[\mathrm{CH}]$

WWW: Overview of how to run an information server ${ }^{\star}$

WWW: WebNews

El WWW permite también el acceso fácil y cómodo a prácticamente todas las bibliotecas del mundo que estén informatizadas. Un primer paso para adentrarse en el campo de las bibliotecas es la utilización de la página del HUMBUL, Humanities Bulletin Board, del Centro de Informatica para Humanidades de la Universidad de Oxford, cuyas entradas son las siguientes ${ }^{33}$ :

${ }^{33}$ EI URL es: http://info.oxac.uk/departments/humanities/lib.htm. 


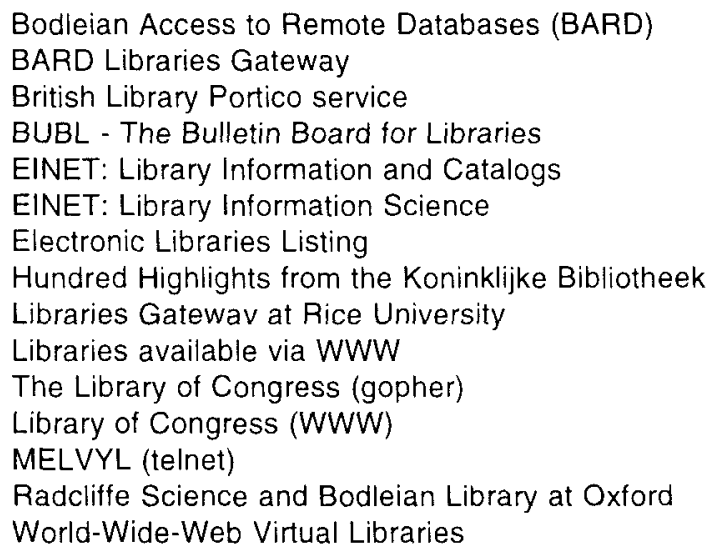

Como puede observarse la palabra biblioteca tiene dos significados: el tradicional como sería el caso de la Biblioteca del Congreso norteamericano ${ }^{34}$ y la informática 0 "virtual», considerada como un conjunto de textos electrónicos que se contienen en Internet. En principio, el acceso a las bibliotecas es público y lo más que se exige es tener una dirección electrónica, pero el acceso a las bibliotecas europeas no es tan eficiente como a las norteamericanas ${ }^{35}$. En el ámbito español la Biblioteca Nacional no se encuentra entre las más avanzadas, pues el 5 de agosto de 1995 solamente se habían fichado informáticamente 506.985 libros y 2 manuscritos como se muestra en el volcado de pantalla situado en la página siguiente ${ }^{36}$.

Es indudable que el uso de los catálogos de las bibliotecas a través del World Wide Web es un elemento más y muy importante en la investigación y en la enseñanza de la Historia Contemporánea tanto para el profesorado como para el alumnado de la Universidad Nacional de Educación a Distancia.

34 Accesible a través de http://lcweb.loc.gov/homepage/lchp.htm.

35 Welsch en su articulo ya citado se queja amargamente: "After experience using TELNET and TN3270 to access online catalogs in Europe, I would reluctantly have to side with the skeptics. My qualms fall into several categories: 1) uncertainty of TELNET access; there are many instancEs when I cannot reach distant catalogs at all except late at night when there is litle competition from oomestic users. At other times the networks or systems are so slow that patience wears thin; 2) systems in European libraries vary so widely in commands that moving from one to the other is a trial; 3) most of the libraries currently on the net are of technical schools whose humanities content is insignificant; 4) despite best efforts, documentation inevitably falls behind changes; and 5) mapping keyboards to foreign, or domestic, interactive services is still imperfect".

${ }_{36}$ A la Biblioteca Nacional puede accederse a través del siguiente URL: gopher://gopher.uii.es/11\%2FCentre\%5FDoc\%2FSpain\%2FBN\%09\%2B application\%2Fgopher $\% 2 B \% 2 D$ menu\%20Es\%5FES. 


\section{ARIADNA \\ El catálogo automatizado de la Biblioteca Nacional contiene las des- cripciones bibliográficas de:

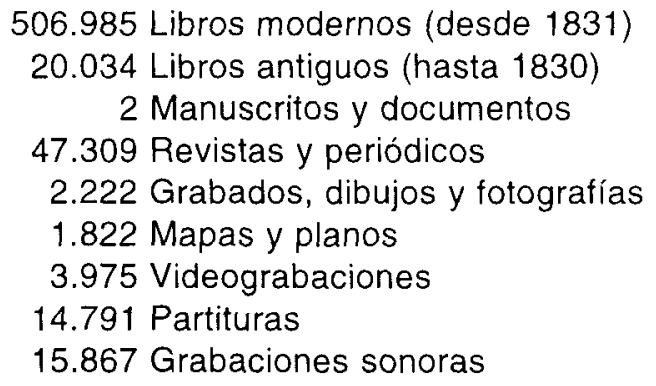

Recuerde que la información que todavía no haya sido introducida en ARIADNA, podrá localizarla en los distintos catálogos de laBiblioteca.

\section{Pulse INTRO Para continuar}

\section{INTERNET Y LA UNED}

De todo lo expuesto hasta aquí se deduce claramente la necesidad de aplicar los medios, recursos dirían los informáticos, que Internet ofrece a los alumnos y profesores del Departamento de Historia Contemporánea de la Universidad Nacional de Educación a Distancia. La aplicación de estos recursos debe concretarse principalmente en la utilización del World Wide Web, porque ofrece la posibilidad de presentar la información no sólo con textos sino también con gráficos, sonidos y animaciones, y del correo electrónico. Dado el inmenso mundo de información que supone el WWW estos recursos pueden servir también a cualquier estudiante o estudioso de la Historia Contemporánea en general y de Historia Contemporánea de España en particular, cumpliéndose así uno de los tradicionales objetivos de la Universidad: difundir el saber a toda la sociedad sin reducirse a un pequeño número de personas o a un territorio minúsculo tal como se expresa en la leyenda del escudo de la UNED, Omnibus Mobilibus Mobilior Sapientia. Es decir, con Internet, y más concretamente con el Web, la Universidad alcanza realmente su categoría de universal. 
Obviamente la primera aplicación de Internet que puede y debe hacerse en el World Wide Web es la creación de unas páginas, sitios, sobre el Departamento, lo que plantea dos problemas: el imperativo, más que categórico, de utilizar un nuevo lenguaje de programación y la necesidad de planificar perfectamente qué es con exactitud lo que se quiere hacer. Ambos problemas aunque fáciles de resolver teóricamente, traen consigo en la práctica un trabajo realmente fatigoso y largo.

Para poder hacer un documento de WWW es preciso conocer y dominar un nuevo lenguaje de programación designado específicamente para crear un documento que tenga extensiones de hipertexto y que, al mismo tiempo, pueda ser leído por los diferentes browser que hay en el WWW. Este lenguaje se llama HTML, acrónimo de HyperText Markup Language. Su esencia consiste en introducir códigos que cualquier browser de WWW pueda reconocer e interpretar con el fin de mostrar en la pantalla del ordenador lo que previamente se había diseñado ${ }^{37}$. Cualquier programa de tipo HTML puede crearse con un procesador de texto que guarde lo escrito en formato ASCII y, de hecho, tratamientos de textos importantes tienen módulos que introducen parte de los códigos de forma más fácil ${ }^{38}$. En principio es más cómodo utilizar algunos de los programas creados exprofeso para la edición de documentos de tipo HTML; comodidad a la que se debe añadir la economía de algunos existentes que facilitan algo la tarea de introducir los innumerables códigos. Uno de los mejores programas, tanto por su sencillez de uso como por su economia es el HTMLWRITER escrito en Visual Basic 3.0 por Kris Nosack ${ }^{39}$.

Una vez que se domina el lenguaje HTML es preciso planificar, una y otra vez, lo que se quiere mostrar en las páginas del WWW. En este caso concreto se trata de mostrar la actividad docente e investigadora del Departamento de Historia Contemporánea de la UNED y en concreto la labor desarrollada en la asignatura de Historia Contemporánea de España.

37 Los códigos más elementales alcanzan el número de treinta y nueve. Una relación de ellos, que naturalmente no intenta ser total, se expone bajo el título de Otmar's List of HTML tags en http://www.cosy.sbg.ac.at./ /endl/tags.html. La enumeración de todos los códigos en Kevin Werbach, The Bare Bones Guide to HTML en http:l/Www.access.digest.net/ vwerbach/barebone.html. La explicación detallada se halla en IAN GRAHAM, Introduction to HTML documentation en http://www. utirc.utoronto.ca/htm/docs/-newhtm//intro.html.

38 La propia multinacional Microsott ha creado una plantilla que funciona sólo con la versión inglesa, francesa y alemana del Word 6.0. Si se quiere utilizar la versión castellana de Word, ya sea $2.0 \circ 6.0$, es preciso conseguir la plantilla, CU_HTML.DOT que ha realizado la Universidad China de Honk-Kong

${ }_{39}$ La economia se basa en su gratuidad, aunque el autor aconseja que se le envie una donación de 10 dólares. Se puede encontrar en http://lal.cs.byu.edu/people/nosack. 
Por tanto, en la página principal habría que informar sobre los siguientes temas:

- Congreso sobre «Historia de la transición y consolidación democrática en España 1976 1986".

- Consejo del Departamento.

- Docencia.

- Publicaciones.

- Lista de Distribución de Historia Contemporánea de España.

- Internet.

Todos estos elementos tendrían la característica de ser hipertexto, de tal forma que cada uno de ellos llevaría a otra página de WWW consiguiéndose el siguiente diagrama de flujo.
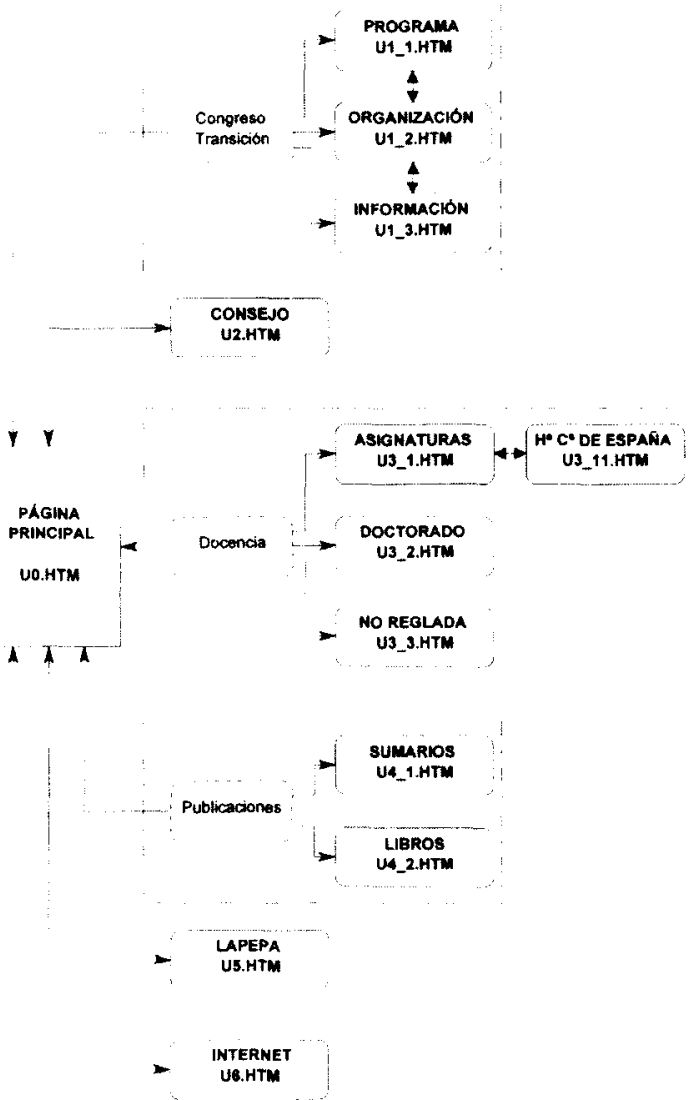
Gráficamente la idea es que aparezcan unas pantallas como las que muestran las figuras que se exponen a continuación, con la advertencia de que la programación informática, y por ende la creación de páginas del WWW, es la versión moderna del mito de Penélope: hacer y deshacer continuamente. Por eso las páginas mostradas en el texto no serán iguales a las que existan en Internet cuando se lea este artículo. Para lograr esta primera página fue preciso escribir un programa de ciento setenta y ocho líneas.

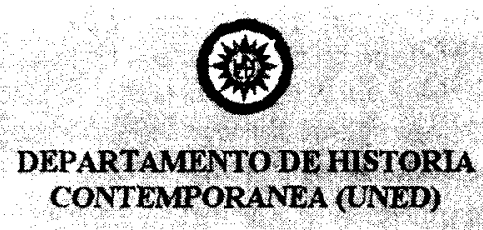

Welcom to the UNED Coniemporiny Ititory Deperment on the World Wide Web. This

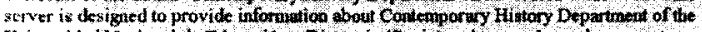
Universidad Nacional de Edxeacibn a Distancin (Spain) yad to noply ready acces to sowe relevent sites for hititoriuns ditribeded acrone the netwonk

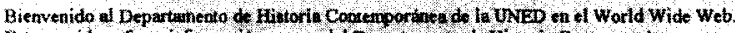

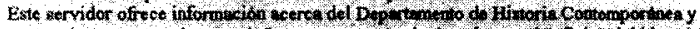

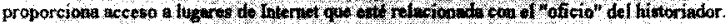

Achulnetate pucde consultar los sigmentes opertedest

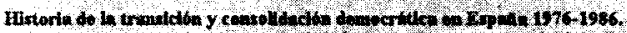

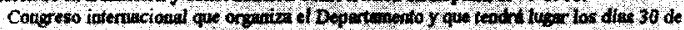
novienbre, 1 y 2 de dicithbre.

0 rograme

a Orgaizacion

0 Inforatios

Consete do Deraxt werong.

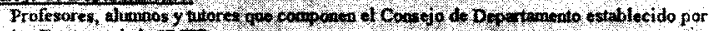
los Estatitos de la onED

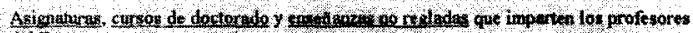
del Departamedio.

Pabliciclenes

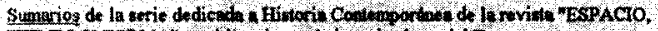

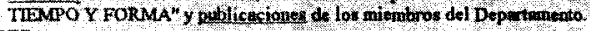
LAPEPA

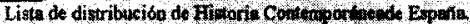
Interant

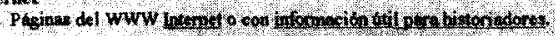

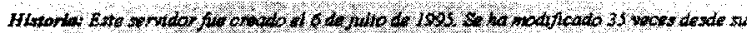

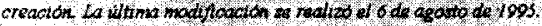

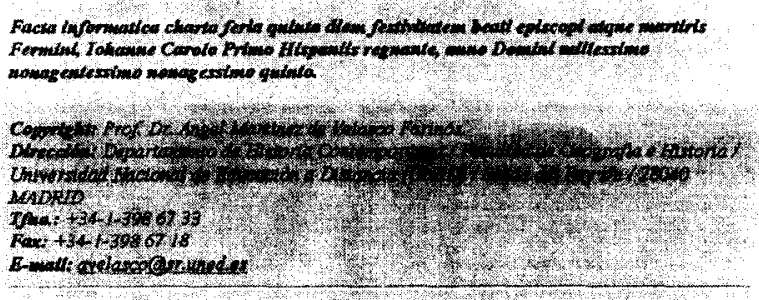


Los hipertextos están subrayados y, en el caso del correo electrónico, avelasco@sr.uned.es, se da paso a una segunda pantalla en la que se puede enviar un mensaje.

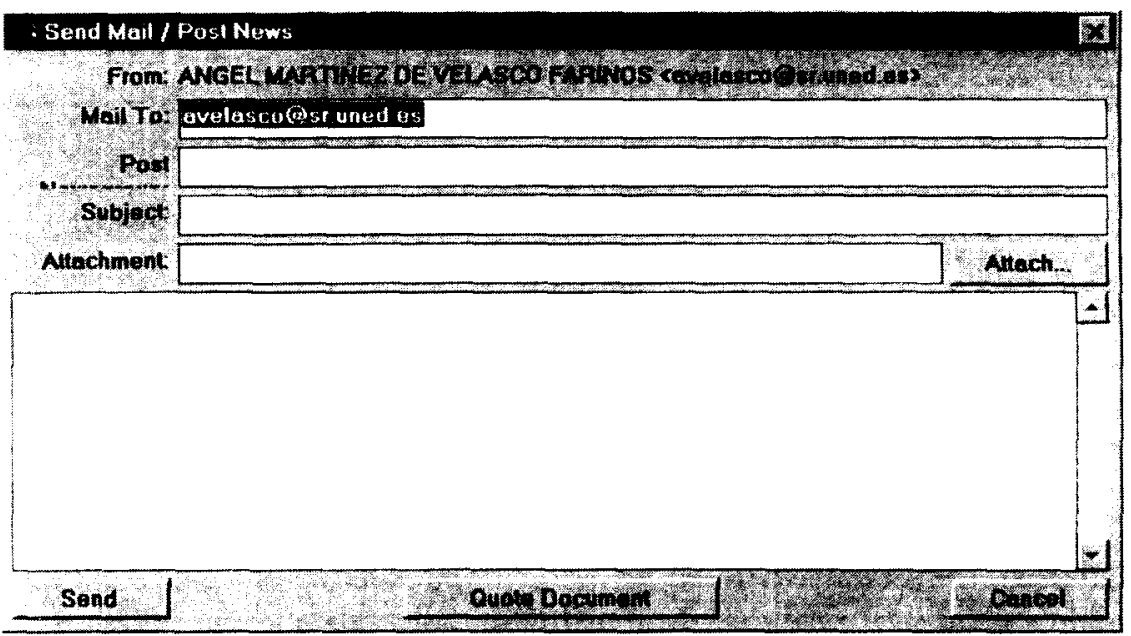

Dentro de estas páginas del World Wide Web sobre el Departamento he dedicado especial atencion a la asignatura de Historia Contemporánea de España no sólo porque es un medio de mostrar ante todo el mundo algo de nuestra Historia sino también por ser la asignatura que actualmente codirijo ${ }^{40}$. Entre los varios apartados que he incluido hay algunos que merecen un comentario. El primero de ellos es el dedicado a los profesores que imparten la asignatura: a primera vista puede causar perplejidad ver a tan elevado número de profesores cuando según el organigrama de la Facultad sólo somos dos los titulares encargados de la asignatura. La labor que desempeñan los tutores en los centros asociados les hace merecedores de ser considerados también como profesores de la asignatura, aunque legalmente sea imposible darles este status jurídico.

40 Se hallan en http://www. uned. es/dept/hist-cont. 
El segundo apartado que interesa destacar es el dedicado a exponer diferentes textos históricos que los alumnos no sólo pueden consultar, sino que pueden llevar a su propio ordenador, - download es el término exacto informático en inglés-, para trabajar con ellos en su propia casa. Para empezar se ha incluído la primera Constitución espanola y el estatuto de Bayona.

En tercer lugar, se ha creado un apartado dedicado a comentarios de texto con una evidente finalidad docente. Estos comentarios no han sido hechos sine ira et studio por los profesores de la asignatura, sino por alumnos durante pasadas pruebas presenciales. Con ello se consigue que sean auténticos paradigmas para los nuevos alumnos. Además se han incluido todos los pequeños trabajos de investigación que los alumnos del curso 1994-1995 han llevado a cabo bajo la dirección de su profesor tutor. Alguno de ellos puede servir a cualquier persona que estudie el mismo o parecido tema; con ello se intenta favorecer el carácter comparativo de la Historia y evitar el defecto tan extendido del localismo.

Finalmente, es preciso destacar que las páginas del WWW no sólo sirven para la docencia de la asignatura de Historia Contemporánea de España, sino también para mostrar la labor investigadora del Departamento. Por ello, se muestran las últimas publicaciones de mis colegas, pero para evitar la relación de amistad, y consecuentemente de subjetividad, que origina el trabajo académico conjunto se ha decidido incluir únicamente la ficha catalográfica y el índice de cada obra.

\section{BIBLIOGRAFIA}

La bibliografía sobre Internet es realmente impresionante. En mayo de 1994 se podían adquirir en el mercado norteamericano los siguientes ciento dieciséis libros de carácter divulgativo ${ }^{41}$.

ABOBA, BERNARD, PC-Internet Connection: TCP Networking for DOS \& Windows. MailCom: Mayo 94, ISBN: 1883979005

Alpha DeVElopment Group, Ten Minute Guide to the Intermet. Alpha Bks IN: Mayo 94, ISBN: 1 567614280

Bang, Steve etc., Intemet Unleashed. HW Sams, US: Marzo 94, ISBN: 067230466 X

BEATTY, GRACE JOELY, Intemet for Windows: Compuserve Edition. Prima Publishing, US: Julio 94, ISBN: 1559585536

41 En realidad se habian publicado ciento cincuenta y ocho libros, pero en la lista adjunta se han eliminado cuarenta y dos que corresponden a reimpresiones. La relación original fue hecha por Carolyn Kotlas del Institute for Academic Technology de la Universidad de Missisipi y puede pedirse a Jonathan Pishney cuya dirección electrónica es jonp.iat@mhs.unc.edu. 
Bellow, LINDA, COUNTING the Intemet: Monitoring Pomographic Transmissions on the Intemet in the Coming Diciembreade; Orig. Ed.. Carnegie: Diciembre 93, ISBN: 0962547638.

BIBB, Introduction to the Internet. Van Nos Reinhold: ISBN: 0442018509.

BLANKEnHorn, Dana, Riding the Internet Highway. New Riders Pub, US: Agosto 93, ISBN: 1 $56205192 X$.

Braun, Eric, Complete Intemet Directory; Orig. Ed.. Fawcett: Noviembre 93, ISBN: 0449908984.

Brown, A.R. (Ed.\}, Implementing Intemet: An Impartial Guide for Banks. Helix Software Consultants: Octubre 91, ISBN: 1873984006.

ButLER, MARZOK, How to Use the Internet. Ziff-Davis P, US: Mayo 94, ISBN: 1562762222.

Cronin, MarzoY J., Doing Business on the intemet. Van Nos Reinhold: Diciembre 94, ISBN: O 442017707.

CUnningham, ANn M. (Ed), Three Views of the Intemet; Orig. Ed.. NFAIS: Marzo 93, ISBN: O 942308417.

CHESWICK, WILLIAM, Firewalls \& Intemet Security: Repelling the Wily Hacker. Addison-Wesley: Enero 95, ISBN: 0201633574.

DeRn, DANIEL P., Internet Guide forNew Users. McGraw: Septiembre 93, ISBN: 007016511.

EDDINGs, JoshuA, How the Intemef Works. Ziff-Davis P, US: Marzo 94, ISBN: 1562761927.

EDDY, ANDY, Internet after Hours: Your Guide to Finding Games Entertainment \& Just Plain Weirdness. Prima Pub: Abril 94, ISBN: 1559585137.

Engle, Marzor E., Internet Connections: A Librarian's Guide to Dial-Up Access \& Use. ALA: Junio 93, ISBN: O 838976778.

EngSt, AdAM C., Internet Starter Kit for the Macintosh. Hayden BK. Co, US: Noviembre 93 ISBN: 1568300646.

ENGST, ADAM, Internet Explorers Kit for Macintosh. Hayden: Abril 94, ISBN: 1568300891.

ENGST, ADAM, Internet Starter Kit for Windows. Pubi: Hayden: Abril 94, ISBN: 1568300948.

ESTRADA, SuSAN, Connecting to the Internet: A Buyer's Guide. O'Reilly \& Associates, US: Marzo 94, ISBN: 1565920619.

FALK, BENNETT, Intemet Roadmap. Sybex: Octubre 93, ISBN: O 782113656.

FRAASE, Michael, Macintosh Internet Tour Guide: The Friendly Handbook for Navigating the Internet. Ventana P, US: Agosto 93, ISBN: 1566040620.

FraAse, MichaEl, PC Intemet Tour Guide: Cruising the Intemet the Easy Way. Ventana Pr: Enero 94, ISBN: 1566040841.

FraAse, MICHAEL, Windows Intemet Tour Guide. Ventana P, US: Marzo 94, ISBN: 1566040817. GafFIN, AdAm, Big Dummy's Guide to the Intemet. MIT Pr: Mayo 94, ISBN: O 262571056.

Gardner, David C., Intemet for Windows: America Online Edition. Prima Publishing, US: Junio 94, ISBN: 1559585544.

GARDNER, JIM, DOS User's Guide to the Intemet. P-H: Noviembre 93, ISBN: 0131068733.

GIBBS, MARzok, Curious about the Internet. Sams: Enero 94, IS8N: 0672304597.

GIBBS, MARZOK, Navigating the Intemet; deluxe ed. Sams: Marzo 94, ISBN: O 672304856.

Gilster, PAll, Internet Navigator: New User's Guide to Network Exploration. Wiley: Octubre 93, ISBN: O 471597821

Godin, SETH, Intemet White Pages, 1994. IDG Bks: Marzo 94, ISBN: 1568843003.

Guide to Internet Protocol Suite-Open Systems Interconnection Coexistence and Migration. XJOPEN Co: Noviembre 91, ISBN: 1872630227.

HAHN, HARLEY, Intemet Yellow Pages. Osborne-McGraw: Febrero 94, ISBN: 0078820235.

HAHN, HARLEY, Intemet: The Complete Reference. McGraw: Octubre 93, ISBN: 0078819806.

HAYDEN Development Staff, Intemet Dictionary. Hayden: Abril 94, ISBN: 1568300956.

Henry, Marzocia, Search Sheets for Opacs on the intemet, 1994. Pubi: Meckler Corp: Septiembre 94, ISBN: O 887369618.

HESLOP, BRENT, Instant Intemet Guide: Hands-On Global Networking. Addison-Wesley: Febrero 94, ISBN: O 201627078.

Hoffman, PaUl, Access the Intemet. Sybex, US: Mayo 94, ISBN: O 782115292.

Hoffman, PaUL, Intemet Instant Reference. Sybex, US: Febrero 94, ISBN: O 782115128.

HouttuIn, JEROEN. Tutorial on Gatewaying Between X400 \& Intemet Mail; Orig. Ed.. Diane Pub: Enero 93, ISBN: O 788100408.

International SRI Staff, Intemet Mailing Lists, 1993 Edition. P-H: Febrero 93, ISBN: O 133279413. International SRI Staff, Intemet: Geffing Started. P-H: Febrero 93, ISBN: 0133279332.

Internet Quick Reference. Que: Marzo 94, ISBN: 1565297482.

Intemet Yellow Pages. New Riders Pub, US: Abril 94, ISBN: 156205306 X. 
ISO and Intemet Management: Coexistence and Interworking. X/OPEN Co: Diciembre 92 ISBN: 1872630677 .

JAFFE, LEE D., Introducing the Intemet Plus: A Trainer's Workshop. Library Solns: Febrero 94 ISBN: 1882208056 .

Jamsa Press Staff, Success with Intemet. Jamsa Pr: Febrero 94, ISBN: 1884133010.

KanTOR, A., Curious About the Intemet?. HW Sams, US: Febrero 94, ISBN: 0672304597.

Kehoe, Brendan P., Zen \& the Ant of the Intemet: A Beginner's Guide; 3rd ed. P.H Gen Ret \& Trav: Enero 94, ISBN: 0131214926.

KENT PETER, Complete Idiot's Guide to intemei Alpha Bks IN: Febrero 94, ISBN: 156761414.

KRoL, Ed. Whole Internet User's Guide \& Catalog; Orig. Ed.. OReilly \& Assocs: Agosto 92 ISBN: 1565920252.

KURSHAN, BaRBARA, Intemet \& More for Kids. Sybex: Junio 94. ISBN: O 782115179

LADNER, SARYN J., Internet \& Special Librarians: Use, Training, \& the Future. SLA: Mayo 93, ISBN: O 871114135

LAMBERT, STEVE, Internet Basics: Your Map to the Global Electronic Super Highway. Random: Octubre 93, ISBN: O 679750231.

Lane, Elizabeth S., Intemet Primer for Information Professionals: A Basic Guide to internet. Networking Technology. Meckler: Enero 93, ISBN: O 88736831 Y.

LAQUEY, TRACY, Intemet Companion Plus: A Beginner's Start-up Kit for Global Networking. Addison-Wesley: Septiembre 93, ISBN: O 201622246.

LEVINE, JOHN, InternetforDummies QuickReference. IDG Bks: Junio 94. ISBN. 150884168.

LEVINE, JOHN, More Internet for Dummies. ¡DG Bks: Junio 94. ISBN: 1568841647.

LYNCH, DANIEL C., Internet System Handbook. Addison-Wesiey: Enero 93, ISSBN: 020156747

MACHOVEC, GEORGE S., Telecommunications, Networking, \& Intemet Giossaiy. Lit into Tech: Noviembre 93, ISBN: O 838976972

MaLAmud, CARL, Exploring the Internet: A Technical Travelogue. P-H: Agosto 92, ISBN: 013 2968983.

MANGER, JASON J., Intemet Guide to Information Services. MCGraw: Septiemibre 94 ISEN: 007 7079051.

MANGER, JASON, Internet Information Guide. McGraw: Marzo 94, ISBN: 0077079051.

MARZOINE, ABriLiL, Intemet: Getting Started; rev. ed.. P.H: Octubre 93, ISBN: 013289596 X.

Maxwell, Christine, Intemet Yellow Pages. New Riders Pub: Marzo 94, ISBN: 156205306 X.

MCCluRE, Charles R., Libraries \& the Intemet. Meckler Corp: Enero 94, ISBN: 0887368247

McCluRe, Charles R., Libraries and the Intemet/NREN: Perspectives, Issues and Challenges Meckler: Diciembre 93, ISBN: O 887368247

Miller, Marzok A., Troubleshooting TCP - IP: Analyzing the Protocols of the intemet; Orig. Ed.. M\&T Bks: Junio 92, ISBN: 1558512683

MILLES, JAMES, Intemet Handbook for Law Librarians. Glanville: Enero 93, ISBN: 087802093.

MITCHELL, SMOOT CARL, Intemet Connection: System Connectivity and Configuration. AddisonWesley: Febrero 94, ISBN: O 201542374

MockaPetris, PaUL, Intemet Technology Series: Domain Name System; 1 st ed. P-H: Mayo 94, ISBN: 0131068652.

Moravec, Hans, Thinking Robots, an Aware Intemet, \& Cyberpunk Librarians: The 1992 LITA President's Program. Lib Info Tech: Septiembre 92, ISBN: O 838976255

MOSTAFA, JAVED etc., Easy intemet Handbook. Teacher Ideas P, US: Mayo 94, ISBN: 0931510 503.

Mostafa, Javed, Easy Intemet Handbook. Hi Willow: Diciembre 94, ISBN: 0931510503.

MOTLEY, LYNNE, Modem U. S. A.: Low Cost \& Free Online Sources for Information, internet. Databases, \& BBSs via Personal Computer \& Modem in 50 States \& Washington, D. C.; 2nd, rev. ed. Allium Pr: Enero 94, ISBN: O $96312336 \mathrm{X}$

NeOU, VIVIAN, Intemet: Domain Administration. Prentice-Hall: Octubre 93, ISBN: 013511180.

NEOU, VIviAN, Internet: Mailing Lists; rev. ed.. P-H: Agosto 93, ISBN: 0132836613.

New Riders Editors, Riding the Internet Highway. New Riders Pub: Junio 93, ISBN: 156205192 X

NEWBy, GREGORY, Directory of Directories on the intemet: A Guide to Information Sources Meckler: Noviembre 93, ISBN: O 887367682.

NorthWestNet Staff, Intemet Passport: NorthWestNet's Guide to Our World Online; 4th ed. NWNet \& NWAcad: Marzo 93, ISBN: O 963528106.

Notess, GREg R., Internet Access Providers: An Intemational Resource Directory. Merkler Corp: Enero 94, ISBN: O 887369332. 
On Intemet 1994: An Intemational Guide to Electronic Joumals, Newsletters, Books, \& Discussion Lists on the Internet. Meckler Corp: Enero 94, ISBN: O 887369294.

PIKE, MARZOY A., Intemet Quickstart. Que: Marzo 94, ISBN: 1565296583.

PotTer, James E., Bridging the Intemet Gap: How to Make the World Your On-Line Oyster! Bridge Lrn Systs: Diciembre 93, ISBN: O 963206982.

QUARTERMAN, JOHN S., E-Mail Companion: Communications Effectively Via the Internet. AddisonWesley: Enero 94, ISBN: O 201406586.

QuaRTERman, John S., InterNet Connection: System Connectivity \& Configuration. Addison Wesley: Enero 94, ISBN: O 201542374.

Que Development Group StafF. HitchHiker's Guide to Intemet; Orig. Ed. Que, ISBN: 1565293533.

Que Development Group, Intemet QuickStart. Que Computer Publishing, US: Marzo 94, ISBN: 1565296583.

Que Development Group, Internet Resource Quick Reference. Que Computer Publishing, US: Marzo 94, ISBN: 1565297482.

Que Development Group, Using the Intemet Book/ Disc Package. Que Computer Publishing, US: Enero 94, ISBN: 1565293533.

RANDALL, NEIL, Teach Yourself the Internet Around the World in 21 Days. Editors: Junio 94, ISBN: O 672305194.

RoBison, DAvID F., All about Intemet FTP Plvs: Leaming \& Teaching to Transfer Files on the Intemet. Library Solns: Febrero 94, ISBN: 1882208064.

Rose, Marzoshall T., Internet Message: Closing the Book with Electronic Mail. Prentice-Hall: Enero 93, ISBN: 0130929417

Rose, MARzoshall T., Simple Book: Introduction to Intemet Management; 2re. of "Simple Book Introduction to Management of TCP/IP Based Intemets". Prentice-Hall: Diciembre 93, ISBN: 0131772546.

SACHS, DAVID, Hands-on Intemet: Beginning Guide for PC Users. P-H Gen Ref \& Trav: Diciembre 93, ISBN: 0130563927.

SANDLER, CORY, Welcome to Internet: From Mystery to Mastery. MIS Press: Noviembre 93, ISBN: 1558283080.

SAvetz, Kevin, Your Internet Consultant: The Faqs of Online Life. Editors: Junio 94, ISBN: O 672305208 .

SMITH, RICHARD, Navigating the Internet. Sams: Julio 93, ISBN: O 672303620.

SOUTER, BILL, Intemet Security: A Brief Survey; Orig. Ed.. Carnegie: Noviembre 93, ISBN: O $96254762 X$.

SRI, International, Intemet Cd; 1 st ed.. P-H: Diciembre 93, ISBN: 0131238523.

SRI, International, Internet Technology Series, Volume 1: Routing; 1st ed. P.H: Mayo 94, ISBN: 0131068571

StILL, Juliole (Ed\}, Intemet Library: Case Studies of Library Intemet Management \& Use. Meckler Corp: Junio 94, ISBN: O 887369650.

Stranglove, M., Handbook of the intemet. Van Nos Reinhold: ISBN: 0442015089.

TENNANT, ROY, Crossing the InterNet Threshold: An Instructional Handbook; Orig. Ed.. Library Solns: Marzo 93, ISBN: 1882208013.

TITTEL, Ed, IntemetAccess Essentials. Acad Pr: Octubre 94, ISBN: 0126913935.

VELJKOV, MARZOK D., Pocket Guides to the Internet Volume: v. 1: Telneting. Meckler: Diciembre 93. ISBN: O $88736943 \mathrm{X}$.

Velukov, Marzok D., Pocket Guides to the Intemet Volume: v.2: Transferring Files with File TrensferProtocol. Meckler: Diciembre 93, ISBN: O 887369448.

VelJKov, MARzoK D., Pocket Guides to the Internet Volume: v.3: Using and Navigating Usenet. Meckler: Diciembre 93, ISBN: O 887369456.

VÉLJKOV, MARZOK D., Pocket Guides to the Intemet Volume: v.4: The Intemet EMail System. Meckler: Diciembre 93, ISBN: O 887369464.

Velukov, MARzoK D., Pocket Guides to the Internet Volume: v.5: Intemet Utilities. Meckler: Diciembre 93, ISBN: O 887369472.

VelJkov, MARzoK D., Pocket Guides to the Intemet Volume: v.6: Terminal Connections. Meckler: Diciembre 93, ISBN: O 887369480.

Vickery, William E. (Ed, Intro. by), Intemet Profiles of intemational Development Contractors \& Grantees, 1989-1990; rev. ed.. Internet NC: Septiembre 89, ISBN: O 922934029.

WIGGINS, RichaRD, Intemet for Everyone: A Guide for Users \& Providers. McGraw: Abril 94, ISBN: 0070670188. 
Wiggins, RichaRD, Intemet for Everyone: A Guide for Users \& Providers. McGraw: Enero 94, ISBN: 0070670196.

WISEBERG, LAURIE S. (Ed), Human Rights Intemet Directory: Eastem Europe \& the U. S. S. R.; Orig. Ed. Human Rights: Abril 87, ISBN: O 939338033.

WYATT, ALLEN L., SUccess with Intemet Language: and Quick Reference. Jamsa P, US: Marzo 94, ISBN: 1884133010.

Este ingente volumen contrasta aún más si se compara con la miseria existente en las publicaciones españolas. La Biblioteca Nacional posee solamente diez libros de los que una gran parte no se puede consultar porque están reservados para los bibliotecarios. Por todo ello me parece más interesante comentar algunos libros fácilmente asequibles en el mercado español que pueden servir de introducción a Internet partiendo del principio de que cualquier publicación impresa en papel sobre el tema, incluso ésta, está pasada de moda en el momento mismo de su publicación puesto que el crecimiento de la red es vertiginoso.

El reciente libro de Javier Guadalajara es una breve y útil introducción siempre y cuando se tenga en cuenta que, quizás por haber sido escrito demasiado deprisa para aprovechar la demanda del mercado, confunde las Autopistas de la Información con Internet ${ }^{42}$. Las autopistas tendrán unas determinadas características técnicas que Internet, hoy por hoy, no posee totalmente ${ }^{43}$. Para ampliar se puede utilizar el libro de Ed Krol titulado Conéctate al mundo de Internet aunque la versión castellana es más bien una versión mejicana por lo que la lectura llega a ser cansina e incluso algunos términos se hacen ininteligibles ${ }^{44}$. En esta obra es interesante la consulta del "Catálogo de recursos de Internet», situado en el último capítulo, para poder hacerse una idea de la información contenida en la red ${ }^{45}$. Las dificultades que se pueden plantear ante los innumerables acrónimos que se emplean se pueden resolver fácilmente con el simpático diccionario publicado por Tom Fahey ${ }^{46}$. Personalmente considero que la más clara y más útil obra para adentrarse en Internet es la guía de la EARN ya citada a comienzos de este artículo junto con una serie de horas de prácticas ante el monitor del ordenador.

${ }^{42}$ Explorando Internet: las autopistas de la información. Madrid, Tower, 1995, 158 págs. ISBN 84-88432-97-6.

${ }^{43}$ Las principales caracterisitcas técnicas de las Autopistas de la Información son la integración de voz, datos e imagenes en tiempo real, el soporte de trasmisión de banda ancha sobre fibra optica y la utilización maslva.

44 México, O'Reilly \& Associates, Inc. 1995, 597 págs.

45 En la edición consultada, la segunda, el catálogo ocupa las páginas 411 a 504.

${ }_{46}$ Net.speak. The Intemet Dictionary. Indianápolis, Hayden Books. 1994 (ISBN 1-56830-095-

6). En la Biblioteca de Humanidades de la UNED posee la siguiente signatura $B H-681.3 d F A H$. 